\title{
CONSTITUTIVE THEORIES FOR THERMOELASTIC SOLIDS IN LAGRANGIAN DESCRIPTION USING GIBBS POTENTIAL
}

\author{
BY \\ YUSSHY MENDOZA
}

B.S. in Mechanical Engineering, Saint Louis University, 2008

Submitted to the graduate degree program in Mechanical Engineering and the Graduate Faculty of the University of Kansas in partial fulfillment of requirements for the degree of Master of Science.

Dr. Karan S. Surana, Thesis Advisor and Chairperson

Dr. Peter W. TenPas

Dr. Bedru Yimer

Date Defended: June 28, 2012 
The Thesis Committee for YUSSHY MENDOZA certifies that this is the approved version of the following thesis:

\section{CONSTITUTIVE THEORIES FOR THERMOELASTIC SOLIDS IN LAGRANGIAN DESCRIPTION USING GIBBS POTENTIAL}

Dr. Karan S. Surana, Thesis Advisor and Chairperson

Date Approved: June 28, 2012 


\section{Abstract}

This thesis presents constitutive theories for finite deformation of homogeneous, isotropic thermoelastic solids in Lagrangian description using Gibbs potential. Since conservation of mass, balance of momenta and the energy equation are independent of the constitution of the matter, the second law of thermodynamics, i.e. entropy inequality, must form the basis for all constitutive theories of the deforming matter to ensure thermodynamic equilibrium during the evolution $[1,2]$. The entropy inequality expressed in terms of Helmholtz free energy is recast in terms of Gibbs potential. The conditions resulting from the entropy inequality expressed in terms of Gibbs potential permit the derivation of constitutive theory for strain tensor in terms of conjugate stress tensor and the constitutive theory for the heat vector. In the work presented here, it is shown that using the conditions resulting from the entropy inequality, the constitutive theory for the strain tensor can be derived using three different approaches: (i) assuming the Gibbs potential to be a function of the invariants of the conjugate stress tensor and then using the conditions resulting from the entropy inequality, (ii) using theory of generators and invariants, and (iii) expanding Gibbs potential in conjugate stress tensor using Taylor series about a known configuration and then using the conditions resulting from the entropy inequality. The constitutive theories resulting from these three approaches are compared for equivalence between them 
as well as their merits and shortcomings. The constitutive theory for the heat vector can also be derived either directly using the conditions resulting from the entropy inequality or using the theory of generators and invariants. The derivation of constitutive theory for heat vector using the theory of generators and invariants with complete set of argument tensors yields a more comprehensive constitutive theory for heat vector. In the work we consider both approaches.

Summaries of the constitutive theories using parallel approaches (as described above) resulting from the entropy inequality expressed in terms of Helmholtz free energy density are also presented and compared for equivalence with the constitutive theories derived using Gibbs potential. 


\section{Acknowledgements}

I would like to express my sincere gratitude to my advisor and committee chair, Dr. Karan Surana (Dean E. Ackers Distinguished Professor of Mechanical Engineering), for his guidance and encouragement in the successful completion of this work. Furthermore, I would like to thank Dr. Peter TenPas and Dr. Bedru Yimer for serving as members of my thesis committee.

This work is dedicated to my family: my parents, who are my role models and source of inspiration; my sister Mirna, who has the brightest of futures ahead of her; and my wife Jenna, without whose love and patience this would not have been possible.

This research was supported by STIR grant from ARO, Mathematical sciences division under the grant number W-911NF-11-1-0471(FED0061541) to the University of Kansas, Lawrence, Kansas and Texas A \& M University, College Station, Texas. The authors are grateful to Dr. Joseph D. Myers, Program Manager, Scientific Computing, ARO. 


\section{Contents}

Abstract

$\begin{array}{ll}\text { Nomenclature } & \text { ix }\end{array}$

1 Introduction 1

2 Entropy inequality expressed in terms of Gibbs potential $\quad 8$

2.1 Conditions resulting from entropy inequality (2.5) for thermoelastic solids, dependent variables in the constitutive theories and their argument tensors . 10

2.1.1 Entropy inequality utilizing $\boldsymbol{\sigma}^{[0]}$ and $\boldsymbol{\varepsilon}$ as a conjugate pair . . . . . 10

2.1.2 Using $\boldsymbol{\sigma}^{[0]}$ and $\boldsymbol{\varepsilon}$ as conjugate measures but the entropy inequality in $\boldsymbol{\sigma}^{[0]}$ and $\boldsymbol{C} \ldots \ldots \ldots \ldots \ldots$

3 Constitutive theories for strain tensor $\varepsilon$ and heat vector $q$ using Gibbs potential

3.1 Constitutive theory for strain tensor $\varepsilon \ldots \ldots \ldots \ldots$

3.1.1 Constitutive theory for $\boldsymbol{\varepsilon}$ using (3.1) and assuming $\Psi$ to be a function of the invariants of $\boldsymbol{\sigma}^{[0]} \ldots \ldots \ldots \ldots$ 
3.1.2 Constitutive theory for $\boldsymbol{\varepsilon}$ using (3.2) and the theory of generators and invariants $[1-22] \ldots \ldots \ldots \ldots \ldots$

3.1.3 Constitutive theory for strain tensor $\boldsymbol{\varepsilon}$ by expanding $\Psi$ in Taylor series in $\boldsymbol{\sigma}^{[0]}$ about a known configuration and then using (3.1) . . 18

3.1.4 Definitions of material coefficients using $\varepsilon^{\varepsilon} \tilde{\alpha}^{i} ; i=0,1,2$ in (3.15)

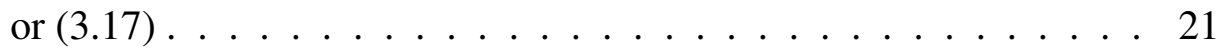

3.2 Constitutive theory for heat vector $\boldsymbol{q} \ldots \ldots \ldots \ldots \ldots$

3.2.1 Constitutive theory for $\boldsymbol{q}$ using entropy inequality $[1,2,8] \ldots 25$

3.2.2 Constitutive theories for $\boldsymbol{q}$ using theory of generators and invariants 26

4 Entropy inequality in terms of Helmholtz free energy density $\Phi$ using conjugate pair $\sigma^{[0]}$ and $\varepsilon$

5 Constitutive theory for stress tensor $\sigma^{[0]}$ and heat vector $q$ using Helmholtz free energy density $\quad 37$

5.1 Constitutive theory for stress tensor $\boldsymbol{\sigma}^{[0]} \ldots \ldots \ldots \ldots \ldots$

5.1.1 Constitutive theory for $\boldsymbol{\sigma}^{[0]}$ using (4.6) and assuming $\Phi$ to be a function of the invariants of $\varepsilon[1,2,8,23] \ldots \ldots \ldots \ldots$

5.1.2 Constitutive theory for $\boldsymbol{\sigma}^{[0]}$ using (4.8) and the theory of generators and invariants $[1-23] \ldots \ldots \ldots \ldots \ldots \ldots$

5.1.3 Constitutive theory for $\boldsymbol{\sigma}^{[0]}$ by expanding $\Phi$ in Taylor series in $\boldsymbol{\varepsilon}$ about a known configuration and then using (4.6) $[1,2,8,23] \quad \ldots \quad 39$

5.1.4 Determination of material coefficients using ${ }^{\sigma} \alpha^{i} ; i=0,1,2$ in (5.2) or $(5.4)[1,2,8,23] \ldots \ldots \ldots \ldots \ldots \ldots$

5.2 Constitutive theory for heat vector $\boldsymbol{q} \ldots \ldots \ldots \ldots \ldots$ 
5.2.1 Constitutive theory for $\boldsymbol{q}$ using entropy inequality $[1,2,8] \ldots 43$

5.2.2 Constitutive theory for $\boldsymbol{q}$ using the theory of generators and invariants $[1,2,8,23] \ldots \ldots \ldots \ldots$

6 Comparison of the constitutive theories resulting from the entropy inequality expressed in terms of Gibbs potential $\Psi$ and Helmholtz free energy density $\Phi \quad 48$

6.1 Constitutive theories for $\boldsymbol{\sigma}^{[0]}$ and $\boldsymbol{\varepsilon} \ldots \ldots \ldots \ldots$

6.1.1 Basic forms resulting from the entropy inequality . . . . . . . . . 49

6.1.2 Constitutive theories for $\boldsymbol{\sigma}^{[0]}$ and $\boldsymbol{\varepsilon}$ from entropy inequalities with material coefficients defined . . . . . . . . . . . . . 49

6.1.3 Constitutive theories for $\boldsymbol{\sigma}^{[0]}$ and $\boldsymbol{\varepsilon}$ from Taylor series expansions of $\Psi$ and $\Phi \ldots \ldots \ldots \ldots \ldots \ldots \ldots \ldots$

6.2 Constitutive theories for heat vector $\boldsymbol{q} \ldots \ldots \ldots \ldots$

6.2.1 Derived directly using conditions resulting from the entropy in-

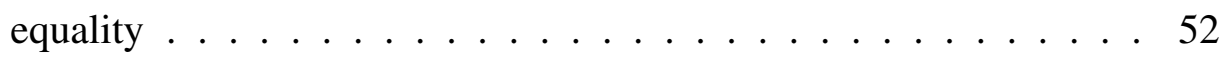

6.2.2 Using the theory of generators and invariants . . . . . . . . 52 


\section{Nomenclature}

$\boldsymbol{\sigma}^{[0]}$ Second Piola-Kirchoff stress tensor

$\boldsymbol{\varepsilon}$ Green's strain tensor

$\theta \quad$ Temperature

$\boldsymbol{q}$ Heat vector

$[J]$ Jacobian of deformation

$\rho_{0} \quad$ Density in the reference configuration

$\Phi \quad$ Helmholtz free energy density

$\eta \quad$ Entropy density

$\Psi \quad$ Gibbs potential

C Cauchy strain tensor

$\boldsymbol{g}$ Temperature gradient

$\underline{\Omega} \quad$ Known configuration 


\section{Chapter 1}

\section{Introduction}

For homogeneous and isotropic thermoelastic solids experiencing finite deformation, the constitutive theories in Lagrangian description are generally derived using the conditions resulting from the entropy inequality expressed in terms of Helmholtz free energy density. If we choose second Piola-Kirchoff stress $\boldsymbol{\sigma}^{[0]}$ and Green's strain tensor $\boldsymbol{\varepsilon}$ as conjugate measures of stress and strain $[1,2,8]$, then this approach yields constitutive theory for $\boldsymbol{\sigma}^{[0]}$ in which $\boldsymbol{\sigma}^{[0]}$ is a function of $\boldsymbol{\varepsilon}$ and the invariants of $\boldsymbol{\varepsilon}$, and the material coefficients are a function of temperature $\theta$ and the invariants of $\boldsymbol{\varepsilon}$ in a chosen known configuration. The simplified constitutive theory for heat vector $\boldsymbol{q}$ derived directly from entropy inequality yields Fourier heat conduction law in which the thermal conductivities can only be a function of temperature $\theta$. The constitutive theories for $\boldsymbol{\sigma}^{[0]}$ and $\boldsymbol{q}$ that are derived using the conditions resulting from the entropy inequality obviously satisfy the second law of thermodynamics. When these constitutive theories are used in the mathematical models derived using conservation of mass, balance of momenta and the first law of thermodynamics, the resulting mathematical model ensures thermodynamic equilibrium of the deforming solid 
during evolution.

An alternative to the entropy inequality expressed in terms of Helmholtz free energy density is to recast the entropy inequality in terms of Gibbs potential and use this form of the entropy inequality and the conditions resulting from it to derive the constitutive theories for the deforming solid during evolution. Since Helmholtz free energy density and Gibbs potential are related, the two forms of the entropy inequalities expressed in terms of Helmholtz free energy density and Gibbs potential are precisely equivalent. However, this equivalence may or may not be preserved in the constitutive theories derived using the two forms of the entropy inequalities and the conditions resulting from these due to the choices of argument tensors and further assumptions employed in the derivations. If we use the condition resulting from the entropy inequality expressed in terms of Gibbs potential, then the constitutive theories for thermoelastic solids result in strain tensor $\boldsymbol{\varepsilon}$ as a function of the stress tensor $\boldsymbol{\sigma}^{[0]}$ and the invariants of $\boldsymbol{\sigma}^{[0]}$. The material coefficients in this constitutive theory are functions of invariants of $\boldsymbol{\sigma}^{[0]}$ and temperature $\theta$ in a known configuration. Derivation of the constitutive theories for homogeneous, isotropic thermoelastic solids experiencing finite deformation using entropy inequality expressed in terms of Gibbs potential is one of the areas of focus in the research presented in this thesis.

The second objective is to compare the constitutive theories derived here with those resulting from the entropy inequality in terms of Helmholtz free energy density, in which case the stress tensor is expressed as a function of the conjugate strain tensor. The work establishes the conditions that ensure equivalence between the constitutive theories derived using Helmholtz free energy density and the Gibbs potential in the entropy inequality. The conditions resulting from the entropy inequality that permit derivation of simple constitutive theory for the heat vector remains unaffected regardless of whether the entropy inequality is 
expressed in terms of Helmholtz free energy density or Gibbs potential. Thus, derivation of the simple constitutive theory for heat vector (such as Fourier heat conduction law) strictly using this condition will obviously yield the same constitutive theory for the heat vector regardless of the choice of Helmholtz free energy density or Gibbs potential. However, the constitutive theories for heat vector derived using the theory of generators and invariants may differ due to the fact that the argument tensors in the two approaches are different. We examine conditions under which these two approaches yield constitutive theories for heat vector that have equivalence.

First, we present a brief literature review related to the constitutive theories that are derived using Gibbs potential. In reference [24] the second law of thermodynamics expressed in Gibbs potential is used to derive constitutive theory for two-phase elastic solids with mass transport for infinitesimal deformation strain tensor. The work in reference [25] is related to application of thermodynamics to thermomechanical fracture and birefringent phenomena in viscoelastic media. For large part this work only considers the second law of thermodynamics expressed in terms of Helmholtz free energy density, but reference is made to the fact that to work with such systems under stressed reference state use of Gibbs free energy is necessary. In reference [26], the authors discuss and present detailed derivations related to chemical equilibrium compositions using Gibbs free energy function. In reference [27] equations of state and constitutive equations are presented for chemical compositions using stress and deformation as conjugate variables in Gibbs free energy. Mechanics and thermodynamics of multiphase flows in porous media including interphase boundaries is presented in reference [28] using entropy inequality expressed in terms of Helmholtz free energy density. The paper presents various relations using Gibbs potential but these are not utilized in the derivations of the constitutive theories. In reference [29] 
Gibbs free energy is used to describe energy balance concepts in the physics of fracture. Using Helmholtz free energy density, the thermodynamic constitutive equations for materials with memory on a material time scale are presented in reference [30]. A formulation for continuum damage mechanics (CDM) for composite materials using Helmholtz free energy density is given in reference [31]. The corresponding form is also presented using Gibbs potential. In reference [32], entropy inequality in terms of Helmholtz free energy density is used to develop constitutive forms and thermodynamic relations for macroscale continuum mechanics for multiphase porous media flows including phase interfaces. Analogous relations using Gibbs potential are not given. Constitutive relations for linear elastic rods using Gibbs potential are given in reference [33].

Authors in reference [34] use entropy inequality in Gibbs potential to derive constitutive theory for strain tensor as a function of second Piola-Kirchoff stress tensor for transformation induced plasticity. Illustrative examples are given using specific forms of Gibbs potential. Constitutive theories for rate dependent dissipative materials using Gibbs potential are given in reference [35]. Stress tensor and temperature are used as argument tensors of the strain tensor. Rate dependent plasticity models are derived from the Gibbs potential in reference [36]. Thermodynamic potentials in linear thermoelasticity are discussed in reference [37]. Entropy inequality is not used explicitly in the derivations of these relations. Thermomechanical formulations for strain gradient plasticity for geomaterials using Gibbs potential are presented in reference [38]. A Gibbs-potential-based formulation for obtaining the response function for a class of viscoelastic materials is presented in reference [39].

From the brief literature review presented here and other works reviewed, we make the following observations regarding the published works. (i) Types of description, i.e. Lagrangian or Eulerian, are not clearly stated in many of the derivations. (ii) Explicit forms 
of the second law of thermodynamics using Gibbs potential in Lagrangian and Eulerian descriptions that form the basic foundation of the derivations of the constitutive theories are rarely given. (iii) For specific applications discussed in the published works related to constitutive theories, choices of dependent variables and their argument tensors and the conditions resulting from the entropy inequality in Gibbs potential that form the basis for deriving constitutive theories are mostly not discussed. (iv) For solid matter almost all constitutive theories are primarily concerned with strain-stress or stress-strain relationships. Compatible constitutive theories for heat vector are never discussed, instead Fourier heat conduction law is almost exclusively used as a constitutive theory for heat vector. (v) Derivations of many constitutive theories (such as $[39,40]$ ) use conditions resulting from the entropy inequality, but the constitutive theories are derived using explicit forms of the Gibbs potential without regards to how this form is arrived at.

\section{Scope of Work}

The work presented in this thesis considers derivations of constitutive theories in Lagrangian description for homogeneous, isotropic thermoelastic solids undergoing finite deformations based on the second law of thermodynamics expressed in terms of Gibbs potential. The entropy inequality is derived in Lagrangian description using Gibbs potential and conjugate stress and strain measures, Second Piola-Kirchoff stress tensor and Green's strain tensor, and is used to determine the possible choice of dependent variables in the constitutive theories. For thermoelastic solids (finite deformation), the possible argument tensors of the Gibbs potential are established. Using Gibbs potential in the entropy in-

equality, conditions are established from which: (i) final choice of dependent variables and their argument tensors is made and (ii) the condition that permit derivations of constitutive 
theories are identified. Using these conditions, constitutive theories for strain tensor $\boldsymbol{\varepsilon}$ are derived in which $\boldsymbol{\varepsilon}$ is a function of $\boldsymbol{\sigma}^{[0]}$ and $\theta$. This is done using three different approaches: (i) assuming the Gibbs potential to be a function of the invariants of the conjugate stress tensor and then using the conditions resulting from the entropy inequality, (ii) using theory of generators and invariants [1-22], and (iii) expanding Gibbs potential in conjugate stress tensor using Taylor series about a known configuration and then using the conditions resulting from the entropy inequality. The constitutive theories resulting from these three approaches are compared for equivalence between them as well as their merits and shortcomings. The constitutive theory for the heat vector is also derived directly using the conditions resulting from the entropy inequality and using the theory of generators and invariants. The derivation of constitutive theory for heat vector using the theory of generators and invariants yields a more comprehensive constitutive theory for heat vector. In the work we consider both approaches.

Summaries of the constitutive theories using parallel approaches (as described above) resulting from the entropy inequality expressed in terms of Helmholtz free energy density $[1,23]$ are also presented and compared for equivalence with those derived using Gibbs potential.

\section{Notations}

We use an over bar to express quantities in the current configuration in Eulerian description, i.e. all quantities with overbars are functions of deformed coordinates $\bar{x}_{i}$ and time $t$. Quantities without an over bar imply Lagrangian description of the quantities in the current configuration, i.e. these are functions of undeformed coordinates $x_{i}$ and time $t$. We use the configuration at time $t=t_{0}=0$, commencement of evolution, to be the reference 
configuration. Thus, $x_{i} ; i=1,2,3$ and $\bar{x}_{i} ; i=1,2,3$ are coordinates of a material point in the reference and current configurations, respectively, both measured in a fixed Cartesian $x$-frame. The present work only considers Lagrangian description, hence all measures are expressed in terms of coordinates of the material points in the undeformed configuration (same as reference configuration in the present work) $x_{i} ; i=1,2,3$ and time $t$. We use $[J]=\left[\frac{\partial\{\bar{x}\}}{\partial\{x\}}\right]$ to be the Jacobian of deformation. We denote $\rho_{0}$ to be the density of the solid matter in the reference configuration, hence it is constant. $\Phi, \theta, \eta$, and $\Psi$ denote the Helmholtz free energy density, temperature, entropy density and Gibbs potential respectively. $\boldsymbol{\sigma}^{[0]}$ is the second Piola-Kirchoff stress tensor. Superscript ' 0 ' is used to signify that it is rate of order zero and the upper case brackets distinguish it from the Cauchy stress tensor $\boldsymbol{\sigma}^{(0)}$ (in contravariant basis). $\boldsymbol{\varepsilon}$ represents Green's strain tensor, a measure of finite strain. $\boldsymbol{\sigma}^{[0]}$ and $\boldsymbol{\varepsilon}$ are a constitutive conjugate pair $[1,2,8]$. Dot on all quantities refers to material derivative. 


\section{Chapter 2}

\section{Entropy inequality expressed in terms of}

\section{Gibbs potential}

We begin with the entropy inequality in Lagrangian description (for finite deformation) expressed in terms of Helmholtz free energy density [1].

$$
\rho_{0}(\dot{\Phi}+\eta \dot{\theta})+\frac{|J|\{q\}^{T}\{g\}}{\theta}-\operatorname{tr}\left(\left[\sigma^{[0]}\right][\dot{\varepsilon}]\right) \leq 0
$$

Dot $(\cdot)$ refers to material derivative, which for Lagrangian description is partial derivative with respect to time. We recall that $\Phi$ and $\Psi$ are related $[1,2,8]$ through

$$
\Psi=\Phi-\frac{1}{\rho_{0}} \operatorname{tr}\left(\left[\sigma^{[0]}\right][\varepsilon]\right)=\Phi-\frac{1}{\rho_{0}} \sigma_{k i}^{[0]} \varepsilon_{i k}
$$

Hence

$$
\dot{\Phi}=\dot{\Psi}+\frac{1}{\rho_{0}} \dot{\sigma}_{k i}^{[0]} \varepsilon_{i k}+\frac{1}{\rho_{0}} \sigma_{k i}^{[0]} \dot{\varepsilon}_{i k}
$$

Substituting from (2.3) into (2.1)

$$
\rho_{0}\left(\dot{\Psi}+\frac{1}{\rho_{0}} \dot{\sigma}_{k i}^{[0]} \varepsilon_{i k}+\frac{1}{\rho_{0}} \sigma_{k i}^{[0]} \dot{\varepsilon}_{i k}+\eta \dot{\theta}\right)+\frac{|J|\{q\}^{T}\{g\}}{\theta}-\sigma_{k i}^{[0]} \dot{\varepsilon}_{i k} \leq 0
$$


or

$$
\rho_{0} \dot{\Psi}+\dot{\sigma}_{k i}^{[0]} \varepsilon_{i k}+\rho_{0} \eta \dot{\theta}+\frac{|J| q_{i} g_{i}}{\theta} \leq 0
$$

Equation (2.5) is the most fundamental form of the entropy inequality expressed in terms of Gibbs potential $\Psi$ and conjugate measures $\boldsymbol{\sigma}^{[0]}$ and $\boldsymbol{\varepsilon}$. An alternate form of (2.5) in $\boldsymbol{\sigma}^{[0]}$ and $\boldsymbol{C}$, Cauchy strain tensor, is sometimes useful and can be derived using

$$
\boldsymbol{\varepsilon}=\frac{1}{2}(\boldsymbol{C}-\boldsymbol{I}) \quad \text { or } \quad \varepsilon_{i k}=\frac{1}{2}\left(C_{i k}-\delta_{i k}\right) \quad ; \quad[C]=[J]^{T}[J]
$$

Thus

$$
\dot{\boldsymbol{\varepsilon}}=\frac{1}{2} \dot{\boldsymbol{C}}
$$

Substituting from (2.7) into (2.1) we obtain

$$
\rho_{0}(\dot{\Phi}+\eta \dot{\theta})+\frac{|J|\{q\}^{T}\{g\}}{\theta}-\frac{1}{2} \operatorname{tr}\left(\left[\sigma^{[0]}\right][\dot{C}]\right) \leq 0
$$

Using (2.2) and (2.6)

$$
\begin{aligned}
\Psi & =\Phi-\frac{1}{\rho_{0}} \operatorname{tr}\left(\left[\sigma^{[0]}\right] \frac{1}{2}([C]-[I])\right) \\
\text { or } \quad \Phi & =\Psi+\frac{1}{2 \rho_{0}}\left(\operatorname{tr}\left(\left[\sigma^{[0]}\right][C]\right)-\operatorname{tr}\left(\left[\sigma^{[0]}\right]\right)\right) \\
\text { or } \quad \Phi & =\Psi+\frac{1}{2 \rho_{0}}\left(\sigma_{k i}^{[0]} C_{i k}-\delta_{i k} \sigma_{k i}^{[0]}\right) \\
\therefore \quad & \dot{\Phi}=\dot{\Psi}+\frac{1}{2 \rho_{0}}\left(\dot{\sigma}_{k i}^{[0]} C_{i k}+\sigma_{k i}^{[0]} \dot{C}_{i k}-\delta_{i k} \dot{\sigma}^{[0]}{ }_{k i}\right)
\end{aligned}
$$

Substituting from (2.10) in (2.8) and rearranging the terms yields

$$
\rho_{0} \dot{\Psi}+\frac{1}{2}\left(C_{i k}-\delta_{i k}\right) \dot{\sigma}_{k i}^{[0]}+\rho_{0} \eta \dot{\theta}+\frac{|J| q_{i} g_{i}}{\theta} \leq 0
$$

Equation (2.11) is also a fundamental form of the entropy inequality expressed in terms of Gibbs potential, $\boldsymbol{\sigma}^{[0]}$ and $\boldsymbol{C}$ with $\boldsymbol{\sigma}^{[0]}$ and $\boldsymbol{\varepsilon}$ as conjugate pair. (2.5) and (2.11) are equivalent. We could have also derived (2.11) using (2.5) and (2.6). 


\subsection{Conditions resulting from entropy inequality (2.5) for thermoelastic solids, dependent variables in the con- stitutive theories and their argument tensors}

\subsubsection{Entropy inequality utilizing $\sigma^{[0]}$ and $\varepsilon$ as a conjugate pair}

We assume that the argument tensors of the Gibbs potential $\Psi$ are $\boldsymbol{\sigma}^{[0]}, \theta$, and $\boldsymbol{g}$. Density in the current configuration is not an argument tensor of the dependent variables due to the fact that $\rho$ is deterministic from $|J|$ and $\rho_{0}$ through the continuity equation.

$$
\Psi=\Psi\left(\boldsymbol{\sigma}^{[0]}, \theta, \boldsymbol{g}\right)
$$

Using (2.12) we can obtain a more explicit form of $\dot{\Psi}$ using chain rule of differentiation.

$$
\dot{\Psi}=\frac{\partial \Psi}{\partial \sigma_{k i}^{[0]}} \dot{\sigma}_{k i}^{[0]}+\frac{\partial \Psi}{\partial g_{i}} \dot{g}_{i}+\frac{\partial \Psi}{\partial \theta} \dot{\theta}
$$

Substituting from (2.13) in (2.5) and regrouping terms

$$
\left(\rho_{0} \frac{\partial \Psi}{\partial \sigma_{k i}^{[0]}}+\varepsilon_{i k}\right) \dot{\sigma}_{k i}^{[0]}+\rho_{0} \frac{\partial \Psi}{\partial g_{i}} \dot{g}_{i}+\rho_{0}\left(\frac{\partial \Psi}{\partial \theta}+\eta\right) \dot{\theta}+\frac{|J| q_{i} g_{i}}{\theta} \leq 0
$$

Inequality (2.14) is a polynomial of degree one in $\dot{\boldsymbol{\sigma}}^{[0]}, \dot{\boldsymbol{g}}$ and $\dot{\theta}$. Since (2.14) must hold for all arbitrary but admissible choices of $\dot{\boldsymbol{\sigma}}^{[0]}, \dot{\boldsymbol{g}}$ and $\dot{\theta}$, this is possible if the following conditions hold:

$$
\begin{aligned}
& \left(\rho_{0} \frac{\partial \Psi}{\partial \sigma_{k i}^{[0]}}+\varepsilon_{i k}\right)=0 \\
& \rho_{0} \frac{\partial \Psi}{\partial g}=0 \\
& \rho_{0}\left(\frac{\partial \Psi}{\partial \theta}+\eta\right)=0 \\
& \frac{|J| q_{i} g_{i}}{\theta} \leq 0
\end{aligned}
$$


Since $\rho_{0}>0,|J|>0$ and $\theta>0$, we can write (2.15) as

$$
\begin{aligned}
& \rho_{0} \frac{\partial \Psi}{\partial \sigma_{k i}^{[0]}}+\varepsilon_{i k}=0 \\
& \frac{\partial \Psi}{\partial g_{i}}=0 \\
& \frac{\partial \Psi}{\partial \theta}+\eta=0 \\
& q_{i} g_{i} \leq 0
\end{aligned}
$$

\section{Remarks:}

From (2.16) we can conclude the following:

1. $\frac{\partial \Psi}{\partial g_{i}}=0$ implies that $\Psi$ is not a function of $\boldsymbol{g}$.

2. $\eta=-\frac{\partial \Psi}{\partial \theta}$ implies that $\eta$ can be derived from $\Psi$ if $\Psi$ is known as a function of temperature, hence $\eta$ cannot be a dependent variable in the constitutive theory.

3. $\varepsilon_{i k}=-\rho_{0} \frac{\partial \Psi}{\partial \sigma_{k i}^{[0]}}$ implies that $\boldsymbol{\varepsilon}$ can be determined from this relationship if $\Psi$ is known as a function of $\boldsymbol{\sigma}^{[0]}$.

4. Since $\Psi$ is not a function of $\boldsymbol{g}$, it implies that $\boldsymbol{\varepsilon}$ and $\eta$ do not depend upon $\boldsymbol{g}$ either.

Based on remarks 1 - 4, we can conclude that $\boldsymbol{\varepsilon}, \Psi$ and $\boldsymbol{q}$ are the only dependent variables in the constitutive theory for solid matter under consideration and their argument tensors are as follows:

$$
\begin{aligned}
& \Psi=\Psi\left(\boldsymbol{\sigma}^{[0]}\left(x_{i}, t\right), \theta\left(x_{i}, t\right)\right) \\
& \boldsymbol{\varepsilon}=\boldsymbol{\varepsilon}\left(\boldsymbol{\sigma}^{[0]}\left(x_{i}, t\right), \theta\left(x_{i}, t\right)\right) \\
& \boldsymbol{q}=\boldsymbol{q}\left(\boldsymbol{\sigma}^{[0]}\left(x_{i}, t\right), \theta\left(x_{i}, t\right), \boldsymbol{g}\left(x_{i}, t\right)\right)
\end{aligned}
$$


At this stage $\boldsymbol{\sigma}^{[0]}$ is an argument tensor of $\boldsymbol{q}$, but can be ruled out later if so warranted by other considerations. From the first equation in (2.16) and the remarks, we have

$$
\varepsilon_{i k}=-\rho_{0} \frac{\partial \Psi}{\partial \sigma_{k i}^{[0]}}
$$

Equation (2.18) forms the basis for deriving constitutive theories in which $\boldsymbol{\varepsilon}$ is a function of $\boldsymbol{\sigma}^{[0]}$. Additionally, $q_{i} g_{i} \leq 0$ can be used to derive the constitutive theory for heat vector. The constitutive theory so derived using (2.12), the last inequality in (2.16) and (2.18) will naturally satisfy the second law of thermodynamics.

\subsubsection{Using $\sigma^{[0]}$ and $\varepsilon$ as conjugate measures but the entropy inequal- ity in $\sigma^{[0]}$ and $\boldsymbol{C}$}

We assume that the argument tensors of Gibbs potential $\Psi$ are $\boldsymbol{\sigma}^{[0]}, \theta$ and $\boldsymbol{g}$ (same as (2.12)) from which we obtain $\dot{\Psi}$ using chain rule of differentiation resulting in (2.13). Substituting (2.13) into (2.11) and rearranging terms we obtain

$$
\left(\rho_{0} \frac{\partial \Psi}{\partial \sigma_{k i}^{[0]}}+\frac{1}{2}\left(C_{i k}-\delta_{i k}\right)\right) \dot{\sigma}_{k i}^{[0]}+\frac{\partial \Psi}{\partial g_{i}} \dot{g}_{i}+\rho_{0}\left(\frac{\partial \Psi}{\partial \theta}+\eta\right) \dot{\theta}+\frac{|J| q_{i} g_{i}}{\theta} \leq 0
$$

Following details presented in section 2.1.1, from (2.19) we can conclude that

$$
\begin{aligned}
& C_{i k}=-2 \rho_{0} \frac{\partial \Psi}{\partial \sigma_{k i}^{[0]}}+\delta_{i k} \\
& \Psi=\Psi\left(\boldsymbol{\sigma}^{[0]}\left(x_{i}, t\right), \theta\left(x_{i}, t\right)\right) \\
& \boldsymbol{C}=\boldsymbol{C}\left(\boldsymbol{\sigma}^{[0]}\left(x_{i}, t\right), \theta\left(x_{i}, t\right)\right) \\
& \boldsymbol{q}=\boldsymbol{q}\left(\boldsymbol{\sigma}^{[0]}\left(x_{i}, t\right), \theta\left(x_{i}, t\right), \boldsymbol{g}\left(x_{i}, t\right)\right)
\end{aligned}
$$


We remark that (2.20) and (2.21) are equivalent to (2.18) and (2.17). It is a matter of choice between the two forms. In all subsequent derivations, we consider (2.18) and (2.17). 


\section{Chapter 3}

\section{Constitutive theories for strain tensor $\varepsilon$}

\section{and heat vector $q$ using Gibbs potential}

In this chapter we present derivations of the constitutive theories for $\boldsymbol{\varepsilon}$ and $\boldsymbol{q}$ using (2.17) and (2.18) based on the entropy inequality using Gibbs potential $\Psi$ and conjugate measures $\boldsymbol{\sigma}^{[0]}$ and $\boldsymbol{\varepsilon}$.

\subsection{Constitutive theory for strain tensor $\varepsilon$}

The constitutive theory for strain tensor $[\varepsilon]$ can be derived based on

$$
\begin{array}{r}
\varepsilon_{i k}=-\rho_{0} \frac{\partial \Psi}{\partial \sigma_{k i}^{[0]}} \\
\text { or } \quad \boldsymbol{\varepsilon}=\boldsymbol{\varepsilon}\left(\boldsymbol{\sigma}^{[0]}, \theta\right)
\end{array}
$$

There are three possible approaches one could take: (i) assuming $\Psi$ to be a function of the invariants of $\boldsymbol{\sigma}^{[0]}$ and $\theta$ and using (3.1), (ii) using (3.2) in conjunction with the theory of generators and invariants [1-22] and (iii) using Taylor series expansion of $\Psi$ in $\boldsymbol{\sigma}^{[0]}$ about 
a known configuration, and then using (3.1). In the work presented here, we derive constitutive theories using all three approaches and examine the resulting constitutive theories from these three approaches to determine when there is equivalence between the resulting theories. Details are presented in the following.

\subsubsection{Constitutive theory for $\varepsilon$ using (3.1) and assuming $\Psi$ to be a function of the invariants of $\sigma^{[0]}$}

In this approach we consider $\Psi$ to be a function of the invariants $I_{\sigma^{[0]}}, I_{\sigma^{[0]}}$ and $\mathbb{I I}_{\sigma^{[0]}}$ (based on characteristic equation for $\boldsymbol{\sigma}^{[0]}$ ) of $\boldsymbol{\sigma}^{[0]}$ and $\theta$ in the current configuration and then use (3.1) to determine the constitutive theory for strain tensor $\boldsymbol{\varepsilon}$.

$$
\Psi=\Psi\left(I_{\sigma^{[0]}}, \mathbb{I}_{\sigma[0]}, \mathbb{I I}_{\sigma^{[0]}}, \theta\right)
$$

in which

$$
\begin{aligned}
& I_{\sigma^{[0]}}=\sigma_{i i}^{[0]}=\operatorname{tr}\left(\boldsymbol{\sigma}^{[0]}\right) \\
& \mathbb{I}_{\sigma^{[0]}}=\frac{1}{2}\left(-\sigma_{k l}^{[0]} \sigma_{l k}^{[0]}+\sigma_{l l}^{[0]} \sigma_{k k}^{[0]}\right) \\
& \mathbb{I I}_{\sigma^{[0]}}=\operatorname{det}\left[\sigma^{[0]}\right]
\end{aligned}
$$

Using (3.3) and (3.1)

$$
[\varepsilon]=-\rho_{0}\left(\frac{\partial \Psi}{\partial I_{\sigma^{[0]}}} \frac{\partial I_{\sigma^{[0]}}}{\partial\left[\sigma^{[0]}\right]}+\frac{\partial \Psi}{\partial \mathbb{I}_{\sigma^{[0]}}} \frac{\partial \mathbb{I}_{\sigma^{[0]}}}{\partial\left[\sigma^{[0]}\right]}+\frac{\partial \Psi}{\partial \mathbb{I I}_{\sigma^{[0]}}} \frac{\partial \mathbb{I I}_{\sigma^{[0]}}}{\partial\left[\sigma^{[0]}\right]}\right)
$$


Using (3.4) - (3.6), it is straightforward to obtain the following

$$
\begin{aligned}
& \frac{\partial I_{\sigma^{[0]}}}{\partial\left[\sigma^{[0]}\right]}=[I] \quad \text { or } \quad \frac{\partial I_{\sigma^{[0]}}}{\partial \sigma_{i j}^{[0]}}=\delta_{i j} \\
& \frac{\partial \mathbb{I}_{\sigma^{[0]}}}{\partial\left[\sigma^{[0]}\right]}=-\left[\sigma^{[0]}\right]+I_{\sigma^{[0]}}[I] \\
& \frac{\partial \mathbb{I I}_{\sigma^{[0]}}}{\partial\left[\sigma^{[0]}\right]}=\mathbb{I I}_{\sigma^{[0]}\left[\sigma^{[0]}\right]^{-1}}
\end{aligned}
$$

Substituting from (3.8) - (3.10) into (3.7)

$$
[\varepsilon]=-\rho_{0}\left(\frac{\partial \Psi}{\partial I_{\sigma^{[0]}}}[I]+\frac{\partial \Psi}{\partial \mathbb{I}_{\sigma^{[0]}}}\left(-\left[\sigma^{[0]}\right]+I_{\sigma^{[0]}}[I]\right)+\frac{\partial \Psi}{\partial \mathbb{I I}_{\sigma^{[0]}}} \mathbb{I I}_{\sigma^{[0]}}\left[\sigma^{[0]}\right]^{-1}\right)
$$

Collecting coefficients of $[I],\left[\sigma^{[0]}\right]$ and $\left[\sigma^{[0]}\right]^{-1}$ in (3.11)

$$
[\varepsilon]=\rho_{0}\left(-\frac{\partial \Psi}{\partial I_{\sigma^{[0]}}}-\frac{\partial \Psi}{\partial \mathbb{I}_{\sigma^{[0]}}} I_{\sigma^{[0]}}\right)[I]+\left(\rho_{0} \frac{\partial \Psi}{\partial \mathbb{I}_{\sigma^{[0]}}}\right)\left[\sigma^{[0]}\right]+\left(-\rho_{0} \frac{\partial \Psi}{\partial \mathbb{I}_{\sigma^{[0]}}} \mathbb{I}_{\sigma^{[0]}}\right)\left[\sigma^{[0]}\right]^{-1}
$$

Let

$$
\begin{aligned}
\varepsilon^{c} \alpha^{0} & =\rho_{0}\left(-\frac{\partial \Psi}{\partial I_{\sigma^{[0]}}}-\frac{\partial \Psi}{\partial \mathbb{I}_{\sigma^{[0]}}} I_{\sigma^{[0]}}\right) \\
\varepsilon^{\alpha} \alpha^{1} & =\rho_{0} \frac{\partial \Psi}{\partial \mathbb{I}_{\sigma^{[0]}}} \\
\varepsilon^{2} \alpha^{2} & =-\rho_{0} \frac{\partial \Psi}{\partial \mathbb{I I}_{\sigma^{[0]}}} \mathbb{I I}_{\sigma^{[0]}}
\end{aligned}
$$

Then

$$
[\varepsilon]={ }^{\varepsilon} \alpha^{0}[I]+{ }^{\varepsilon} \alpha^{1}\left[\sigma^{[0]}\right]+{ }^{\varepsilon} \alpha^{2}\left[\sigma^{[0]}\right]^{-1}
$$

$\left[\sigma^{[0]}\right]^{-1}$ in (3.14) can be substituted in terms of $[I],\left[\sigma^{[0]}\right],\left[\sigma^{[0]}\right]^{2}$ and the invariants of $\left[\sigma^{[0]}\right]$ using the Hamilton Cayley theorem [1] to obtain

$$
[\varepsilon]={ }^{\varepsilon} \tilde{\alpha}^{0}[I]+{ }^{\varepsilon} \tilde{\alpha}^{1}\left[\sigma^{[0]}\right]+{ }^{\varepsilon} \tilde{\alpha}^{2}\left[\sigma^{[0]}\right]^{2}
$$

in which ${ }^{\varepsilon} \tilde{\alpha}^{i} ; i=0,1,2$ are functions of ${ }^{\varepsilon} \alpha^{i} ; i=0,1,2$ and the invariants $I_{\sigma[0]}, \mathbb{I}_{\sigma^{[0]}}$ and $\mathbb{I I I}_{\sigma^{[0]}}$ and temperature $\theta$. Thus, the coefficients ${ }^{\varepsilon} \tilde{\alpha}^{i}={ }^{\varepsilon} \tilde{\alpha}^{i}\left(I_{\sigma^{[0]}}, I_{\sigma^{[0]}}, I I_{\sigma^{[0]}}, \theta\right) ; i=0,1,2$. 
We note that $\rho_{0}$ is in the reference configuration (hence, fixed or constant) but $I_{\sigma^{[0]}}, I_{\sigma^{[0]}}$, $I_{\sigma^{[0]}}$ and $\theta$ are in the current configuration. The constitutive theory (3.15) is not usable yet due to the fact that ${ }^{\varepsilon} \tilde{\alpha}^{i} ; i=0,1,2$ are functions of unknown deformation in the current configuration, hence not deterministic. We postpone further details of determining material coefficients using (3.15) until a later section. However, (3.15) is the fundamental form of the constitutive theory for $[\varepsilon]$ as a function of $\left[\sigma^{[0]}\right]$.

\subsubsection{Constitutive theory for $\varepsilon$ using (3.2) and the theory of generators and invariants [1-22]}

Consider (3.2), i.e.

$$
[\varepsilon]=\left[\varepsilon\left(\left[\sigma^{[0]}\right], \theta\right)\right]
$$

$[\varepsilon]$ is a symmetric tensor of rank two whose argument tensors are $\left[\sigma^{[0]}\right]$, a symmetric tensor of rank two, and $\theta$, a tensor of rank zero. Based on the theory of generators and invariants, $[\varepsilon]$ can be expressed as a linear combination of $[I]$ and the combined generators of its argument tensors which in this case are the generators of $[\varepsilon]$ that are symmetric tensors of rank two. Between the argument tensors $\left[\sigma^{[0]}\right]$ and $\theta$, the combined generators that are symmetric tensors of rank two are $\left[\sigma^{[0]}\right]$ and $\left[\sigma^{[0]}\right]^{2}$. Hence, $[\varepsilon]$ can be expressed as a linear combination of $[I],\left[\sigma^{[0]}\right]$ and $\left[\sigma^{[0]}\right]^{2}$. Using the same coefficients in the linear combination as appear in (3.14) we can write

$$
[\varepsilon]={ }^{\varepsilon} \tilde{\alpha}^{0}[I]+{ }^{\varepsilon} \tilde{\alpha}^{1}\left[\sigma^{[0]}\right]+{ }^{\varepsilon} \tilde{\alpha}^{2}\left[\sigma^{[0]}\right]^{2}
$$


in which the coefficients ${ }^{\varepsilon} \tilde{\alpha}^{i} ; i=0,1,2$ are functions of the combined invariants of argument tensors and $\theta$ in the current configuration.

$$
{ }^{\varepsilon} \tilde{\alpha}^{i}={ }^{\varepsilon} \tilde{\alpha}^{i}\left(I_{\sigma^{[0]}}, I_{\sigma[0]}, I_{\sigma[0]}, \theta\right) \quad ; \quad i=0,1,2
$$

We note that (3.17) is same as (3.15) from the first approach in section 3.1.1 with the same definitions of the coefficients. Thus, the remarks made regarding the coefficients in section 3.1.1 hold here as well. When using the theory of generators and invariants it is easier to use principal invariants $i_{\sigma^{[0]}}, i i_{\sigma^{[0]}}$ and $i i i_{\sigma^{[0]}}$ instead of $I_{\sigma^{[0]}}, \mathbb{I}_{\sigma^{[0]}}$ and $\mathbb{I I}_{\sigma^{[0]}}$ in (3.18). Since the two sets of invariants are related [1], the final outcome remains the same as in section 3.1.1.

\subsubsection{Constitutive theory for strain tensor $\varepsilon$ by expanding $\Psi$ in Taylor series in $\boldsymbol{\sigma}^{[0]}$ about a known configuration and then using (3.1)}

We consider $\Psi=\Psi\left(\boldsymbol{\sigma}^{[0]}, \theta\right)$ and expand $\Psi$ in $\boldsymbol{\sigma}^{[0]}$ using Taylor series about a known configuration $\underline{\Omega}$

$$
\begin{aligned}
\Psi= & \left.\Psi\right|_{\underline{\Omega}}+\left.\frac{\partial \Psi}{\partial \sigma_{i j}^{[0]}}\right|_{\underline{\Omega}}\left(\sigma_{i j}^{[0]}-\left(\sigma_{i j}^{[0]}\right)_{\underline{\Omega}}\right)+ \\
& \left.\frac{1}{2 !} \frac{\partial^{2} \Psi}{\partial \sigma_{i j}^{[0]} \partial \sigma_{k l}^{[0]}}\right|_{\underline{\Omega}}\left(\sigma_{i j}^{[0]}-\left(\sigma_{i j}^{[0]}\right)_{\underline{\Omega}}\right)\left(\sigma_{k l}^{[0]}-\left(\sigma_{k l}^{[0]}\right)_{\underline{\Omega}}\right)+ \\
& \left.\frac{1}{3 !} \frac{\partial^{3} \Psi}{\partial \sigma_{i j}^{[0]} \partial \sigma_{k l}^{[0]} \partial \sigma_{p q}^{[0]}}\right|_{\underline{\Omega}}\left(\sigma_{i j}^{[0]}-\left(\sigma_{i j}^{[0]}\right)_{\underline{\Omega}}\right)\left(\sigma_{k l}^{[0]}-\left(\sigma_{k l}^{[0]}\right)_{\underline{\Omega}}\right)\left(\sigma_{p q}^{[0]}-\left(\sigma_{p q}^{[0]}\right)_{\underline{\Omega}}\right)+\ldots
\end{aligned}
$$


Let

$$
\begin{aligned}
& \left.\Psi\right|_{\underline{\Omega}}=C \\
& \frac{\partial \Psi}{\left.\partial \sigma_{i j}^{[0]}\right|_{\underline{\Omega}}}=C_{i j} \\
& \frac{\partial^{2} \Psi}{\left.\partial \sigma_{i j}^{[0]} \partial \sigma_{k l}^{[0]}\right|_{\underline{\Omega}}}=\hat{C}_{i j k l} \\
& \left.\frac{\partial^{3} \Psi}{\partial \sigma_{i j}^{[0]} \partial \sigma_{k l}^{[0]} \partial \sigma_{p q}^{[0]}}\right|_{\underline{\Omega}}=\tilde{C}_{i j k l p q}
\end{aligned}
$$

The coefficients in (3.20) are defined in a known configuration $\underline{\Omega}$. Using (3.20) in (3.19)

$$
\begin{aligned}
\Psi= & C+C_{i j}\left(\sigma_{i j}^{[0]}-\left(\sigma_{i j}^{[0]}\right)_{\underline{\Omega}}\right)+\frac{1}{2 !} \hat{C}_{i j k l}\left(\sigma_{i j}^{[0]}-\left(\sigma_{i j}^{[0]}\right)_{\underline{\Omega}}\right)\left(\sigma_{k l}^{[0]}-\left(\sigma_{k l}^{[0]}\right)_{\underline{\Omega}}\right)+ \\
& \frac{1}{3 !} \tilde{C}_{i j k l p q}\left(\sigma_{i j}^{[0]}-\left(\sigma_{i j}^{[0]}\right)_{\underline{\Omega}}\right)\left(\sigma_{k l}^{[0]}-\left(\sigma_{k l}^{[0]}\right)_{\underline{\Omega}}\right)\left(\sigma_{p q}^{[0]}-\left(\sigma_{p q}^{[0]}\right)_{\underline{\Omega}}\right)+\ldots
\end{aligned}
$$

Differentiating $\Psi$ with respect to $\boldsymbol{\sigma}^{[0]}$ and using (3.1) and noting that partial derivatives of the coefficients in (3.20) with respect to $\boldsymbol{\sigma}^{[0]}$ are zero and

$$
\begin{aligned}
& \frac{\partial}{\partial \sigma_{m n}^{[0]}}\left(\sigma_{i j}^{[0]}-\left(\sigma_{i j}^{[0]}\right)_{\underline{\Omega}}\right)=\frac{\partial \sigma_{i j}^{[0]}}{\partial \sigma_{m n}^{[0]}}=\delta_{i m} \delta_{j n} \\
& \frac{\partial}{\partial \sigma_{m n}^{[0]}}\left(\left(\sigma_{i j}^{[0]}-\left(\sigma_{i j}^{[0]}\right)_{\underline{\Omega}}\right)\left(\sigma_{k l}^{[0]}-\left(\sigma_{k l}^{[0]}\right)_{\Omega}\right)\right) \\
& =\delta_{i m} \delta_{j n}\left(\sigma_{k l}^{[0]}-\left(\sigma_{k l}^{[0]}\right)_{\underline{\Omega}}\right)+\delta_{k m} \delta_{l n}\left(\sigma_{i j}^{[0]}-\left(\sigma_{i j}^{[0]}\right)_{\underline{\Omega}}\right) \\
& \frac{\partial}{\partial \sigma_{m n}^{[0]}}\left(\left(\sigma_{i j}^{[0]}-\left(\sigma_{i j}^{[0]}\right)_{\Omega}\right)\left(\sigma_{k l}^{[0]}-\left(\sigma_{k l}^{[0]}\right)_{\Omega}\right)\left(\sigma_{p q}^{[0]}-\left(\sigma_{p q}^{[0]}\right)_{\Omega}\right)\right) \\
& =\delta_{i m} \delta_{j n}\left(\sigma_{k l}^{[0]}-\left(\sigma_{k l}^{[0]}\right)_{\underline{\Omega}}\right)\left(\sigma_{p q}^{[0]}-\left(\sigma_{p q}^{[0]}\right)_{\underline{\Omega}}\right)+ \\
& \delta_{k m} \delta_{l n}\left(\sigma_{i j}^{[0]}-\left(\sigma_{i j}^{[0]}\right)_{\underline{\Omega}}\right)\left(\sigma_{p q}^{[0]}-\left(\sigma_{p q}^{[0]}\right)_{\underline{\Omega}}\right)+ \\
& \delta_{p m} \delta_{q n}\left(\sigma_{i j}^{[0]}-\left(\sigma_{i j}^{[0]}\right)_{\underline{\Omega}}\right)\left(\sigma_{k l}^{[0]}-\left(\sigma_{k l}^{[0]}\right)_{\underline{\Omega}}\right)
\end{aligned}
$$

We obtain (3.26) if we substitute from (3.22) - (3.25) in (3.1). In doing so (i) we collect all terms in configuration $\underline{\Omega}$, (ii) we define the coefficients of $\left[\sigma^{[0]}\right]$ and $\left[\sigma^{[0]}\right]^{2}$ (those that are defined in configuration $\underline{\Omega}$ ) in the current configuration and (iii) we use symmetry of the 
coefficients, i.e. $\hat{C}_{m n i j}=\hat{C}_{i j m n}$, etc.

$$
\varepsilon_{m n}=\left(\varepsilon_{m n}^{0}\right)_{\underline{\Omega}}+\left(\underline{\underline{C}}_{m n i j}\right)_{\underline{\Omega}} \sigma_{i j}^{[0]}+\left(\underline{\underline{\tilde{C}}}_{m n i j k l}\right)_{\underline{\Omega}} \sigma_{i j}^{[0]} \sigma_{k l}^{[0]}+\ldots
$$

\section{Remarks:}

1. We note that (3.26) and (3.15), (3.17) are similar in the sense that all these three forms contain exactly the same tensors on the left and right side of the equality that are in the current configuration.

2. In (3.15) and (3.17) the coefficients ${ }^{\varepsilon} \tilde{\alpha}^{i} ; i=0,1,2$ are in the current configuration, whereas in (3.26) the coefficients are in the known configuration $\underline{\Omega}$. Hence, constitutive theory (3.26) is quite different compared to (3.15) or (3.17).

3. Based on the derivations given in sections 3.1.1 and 3.1.2, it is clear that the Taylor series expansion in (3.19) must be limited up to the cubic terms in $\boldsymbol{\sigma}^{[0]}$. Inclusion of further higher degree terms in $\boldsymbol{\sigma}^{[0]}$ is non-physical as it is not supported by the derivations in sections 3.1.1 and 3.1.2 that are strictly based on the entropy inequality.

4. From Taylor series expansion it is clear that the coefficients in (3.26) are functions of $\boldsymbol{\sigma}^{[0]}$ and $\theta$ in a known configuration $\underline{\Omega}$, whereas the coefficients ${ }^{\varepsilon} \tilde{\alpha}^{i}$ in (3.15) and (3.17) are functions of invariants of $\left[\sigma^{[0]}\right]$ and $\theta$ in the current configuration. The coefficients in (3.26) are in fact material coefficients whereas the coefficients in (3.15) and (3.17) are yet to be defined using ${ }^{\varepsilon} \tilde{\alpha}^{i} ; i=01,2$.

5. The issue of whether (3.26) is superior over (3.15) or (3.17) and vice versa can only be addressed after we determine the material coefficients using $\varepsilon^{\varepsilon} \tilde{\alpha}^{i} ; i=0,1,2$. We present details in the following section. 
6. For homogeneous isotropic solid matter the coefficients in (3.26) can be simplified [1].

\subsubsection{Definitions of material coefficients using ${ }^{\varepsilon} \tilde{\alpha}^{i} ; i=0,1,2$ in (3.15) or $(3.17)$}

Consider

$$
[\varepsilon]={ }^{\varepsilon} \tilde{\alpha}^{0}[I]+{ }^{\varepsilon} \tilde{\alpha}^{1}\left[\sigma^{[0]}\right]+{ }^{\varepsilon} \tilde{\alpha}^{2}\left[\sigma^{[0]}\right]^{2}
$$

We consider ${ }^{\varepsilon} \tilde{\alpha}^{i}$ to be functions of $I_{\sigma^{[0]}}, I_{\sigma^{[0]}}$ and $I I_{\sigma^{[0]}}$ (as opposed to principal invariants) and $\theta$.

$$
{ }^{\varepsilon} \tilde{\alpha}^{i}={ }^{\varepsilon} \tilde{\alpha}^{i}\left(I_{\sigma^{[0]}}, I_{\sigma^{[0]}}, I_{\sigma^{[0]}}, \theta\right)
$$

We can expand each ${ }^{\varepsilon} \tilde{\alpha}^{i}$ in Taylor series in $I_{\sigma^{[0]}}, I_{\sigma^{[0]}}, I I_{\sigma^{[0]}}$ and $\theta$ about a known configuration $\underline{\Omega}$. We retain only up to linear terms in the invariants of $\left[\sigma^{[0]}\right]$ and $\theta$ in the Taylor series expansion. To make the derivation compact we define

$$
{ }_{\sim}^{\varepsilon} I^{1}=I_{\sigma[0]} \quad ; \quad{ }_{\sim}^{\varepsilon} I^{2}=\mathbb{I}_{\sigma^{[0]}} \quad ; \quad{ }^{\varepsilon} \underline{\sim}^{3}=\mathbb{I I}_{\sigma^{[0]}}
$$

Using the notation in (3.29), we can write

$$
{ }^{\varepsilon} \tilde{\alpha}^{i}=\left.{ }^{\varepsilon} \tilde{\alpha}^{i}\right|_{\underline{\Omega}}+\left.\sum_{j=1}^{3} \frac{\partial \tilde{\alpha}^{\varepsilon}}{\partial \tilde{\alpha}^{\varepsilon}}\right|_{\underline{\Omega}}\left({ }_{\underline{\sim}}^{\varepsilon} I^{j}-\left({ }^{\varepsilon} I^{j}\right)_{\underline{\Omega}}\right)+\left.\frac{\partial^{\varepsilon} \tilde{\alpha}^{i}}{\partial \theta}\right|_{\underline{\Omega}}\left(\theta-\theta_{\underline{\Omega}}\right) \quad ; \quad i=0,1,2
$$

Substituting from (3.30) into (3.27)

$$
\begin{aligned}
& {[\varepsilon]=\left(\left.{ }^{\varepsilon} \tilde{\alpha}^{0}\right|_{\underline{\Omega}}+\left.\sum_{j=1}^{3} \frac{\partial^{\varepsilon} \tilde{\alpha}^{0}}{\partial^{\varepsilon} I_{\sim}^{j}}\right|_{\underline{\Omega}}\left({ }_{\sim}^{\varepsilon} I^{j}-\left({ }^{\varepsilon} I^{j}\right)_{\underline{\Omega}}\right)+\left.\frac{\partial^{\varepsilon} \tilde{\alpha}^{0}}{\partial \theta}\right|_{\underline{\Omega}}\left(\theta-\theta_{\underline{\Omega}}\right)\right)[I]+} \\
& \left(\left.\tilde{\alpha}^{\varepsilon}\right|_{\underline{\Omega}}+\left.\sum_{j=1}^{3} \frac{\partial^{\varepsilon} \tilde{\alpha}^{1}}{\partial^{\varepsilon} I_{\sim}^{j}}\right|_{\underline{\Omega}}\left({ }^{\varepsilon} I^{j}-\left({ }^{\varepsilon} I^{j}\right)_{\underline{\Omega}}\right)+\left.\frac{\partial^{\varepsilon} \tilde{\alpha}^{1}}{\partial \theta}\right|_{\underline{\Omega}}\left(\theta-\theta_{\underline{\Omega}}\right)\right)\left[\sigma^{[0]}\right]+ \\
& \left(\left.\tilde{\alpha}^{2}\right|_{\underline{\Omega}}+\left.\sum_{j=1}^{3} \frac{\partial^{\varepsilon} \tilde{\alpha}^{2}}{\partial^{\varepsilon} I_{\sim}^{j}}\right|_{\underline{\Omega}}\left({ }_{\sim}^{\varepsilon} I^{j}-\left({ }_{\sim}^{\varepsilon} I^{j}\right)_{\underline{\Omega}}\right)+\left.\frac{\partial^{\varepsilon} \tilde{\alpha}^{2}}{\partial \theta}\right|_{\underline{\Omega}}\left(\theta-\theta_{\underline{\Omega}}\right)\right)\left[\sigma^{[0]}\right]^{2}
\end{aligned}
$$




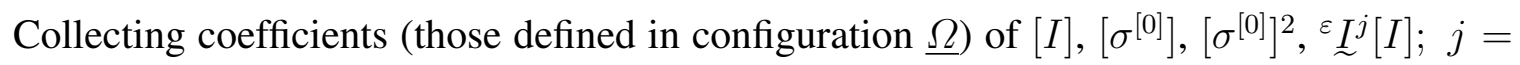
$1,2,3,{ }^{\varepsilon} \underline{\sim}^{j}\left[\sigma^{[0]}\right] ; j=1,2,3,{ }^{\varepsilon} \underline{\sim}^{j}\left[\sigma^{[0]}\right]^{2} ; j=1,2,3,\left(\theta-\theta_{\underline{\Omega}}\right)[I],\left(\theta-\theta_{\underline{\Omega}}\right)\left[\sigma^{[0]}\right]$, and $(\theta-$ $\left.\theta_{\underline{\Omega}}\right)\left[\sigma^{[0]}\right]^{2}$, we can write the following using (3.31).

$$
\begin{aligned}
& \left.[\varepsilon]=\left(\left.\varepsilon \tilde{\alpha}^{0}\right|_{\underline{\Omega}}-\left.\sum_{j=1}^{3} \frac{\partial^{\varepsilon} \tilde{\alpha}^{0}}{\partial^{\varepsilon} I_{\sim}^{j}}\right|_{\underline{\Omega}} ^{\left({ }^{\varepsilon} I_{\sim}^{j}\right.}\right)_{\underline{\Omega}}\right)[I]+ \\
& \left.\left(\left.\tilde{\alpha}^{\varepsilon}\right|_{\underline{\Omega}}-\left.\sum_{j=1}^{3} \frac{\partial^{\varepsilon} \tilde{\alpha}^{1}}{\partial^{\varepsilon} I_{\sim}^{j}}\right|_{\underline{\Omega}} ^{\left({ }^{\varepsilon}\right.} I^{j}\right)_{\underline{\Omega}}\right)\left[\sigma^{[0]}\right]+ \\
& \left(\left.\tilde{\alpha}^{\varepsilon}\right|_{\underline{\Omega}}-\left.\sum_{j=1}^{3} \frac{\partial^{\varepsilon} \tilde{\alpha}^{2}}{\partial^{\varepsilon} I_{\sim}^{j}}\right|_{\underline{\Omega}}\left({ }^{\varepsilon} I^{j}\right)_{\underline{\Omega}}\right)\left[\sigma^{[0]}\right]^{2}+ \\
& \sum_{j=1}^{3} \frac{\partial^{\varepsilon} \tilde{\alpha}^{0}}{\partial^{\varepsilon} I_{\sim}^{j}}\left({ }_{\sim}^{\varepsilon} I_{\sim}^{j}[I]\right)+\sum_{j=1}^{3} \frac{\partial^{\varepsilon} \tilde{\alpha}^{1}}{\partial^{\varepsilon} I_{\sim}^{j}}\left({ }_{\sim}^{\varepsilon} I^{j}\left[\sigma^{[0]}\right]\right)+\sum_{j=1}^{3} \frac{\partial^{\varepsilon} \tilde{\alpha}^{2}}{\partial^{\varepsilon} I_{\sim}^{j}}\left({ }_{\sim}^{\varepsilon} I_{\sim}^{j}\left[\sigma^{[0]}\right]^{2}\right)+ \\
& \left.\frac{\partial^{\varepsilon} \tilde{\alpha}^{0}}{\partial \theta}\right|_{\underline{\Omega}}\left(\left(\theta-\theta_{\underline{\Omega}}\right)[I]\right)+\left.\frac{\partial^{\varepsilon} \tilde{\alpha}^{1}}{\partial \theta}\right|_{\underline{\Omega}}\left(\left(\theta-\theta_{\underline{\Omega}}\right)\left[\sigma^{[0]}\right]\right)+\left.\frac{\partial^{\varepsilon} \tilde{\alpha}^{2}}{\partial \theta}\right|_{\underline{\Omega}}\left(\left(\theta-\theta_{\underline{\Omega}}\right)\left[\sigma^{[0]}\right]^{2}\right)
\end{aligned}
$$

Let us define

$$
\begin{aligned}
& \left.\left.\varepsilon_{0}\right|_{\underline{\Omega}}=\left.{ }^{\varepsilon} \tilde{\alpha}^{0}\right|_{\underline{\Omega}}-\left.\sum_{j=1}^{3} \frac{\partial^{\varepsilon} \tilde{\alpha}^{0}}{\partial^{\varepsilon} I_{\sim}^{j}}\right|_{\underline{\Omega}} ^{\left({ }^{\varepsilon}\right.} I_{\sim}^{j}\right)_{\underline{\Omega}} \\
& {\underset{\sim}{a}}_{a_{j}}=\left.\frac{\partial^{\varepsilon} \tilde{\alpha}^{0}}{\partial^{\varepsilon} I_{\sim}^{j}}\right|_{\underline{\Omega}} \quad ; \quad j=1,2,3 \\
& { }^{\varepsilon} b_{i}=\left.{ }^{\varepsilon} \tilde{\alpha}^{i}\right|_{\underline{\Omega}}-\left.\sum_{j=1}^{3} \frac{\partial^{\varepsilon} \tilde{\alpha}^{i}}{\partial^{\varepsilon} I_{\sim}^{j}}\right|_{\underline{\Omega}} \quad ; \quad i=1,2 \\
& { }_{\sim}^{\varepsilon} c_{1 j}=\left.\frac{\partial^{\varepsilon} \tilde{\alpha}^{1}}{\partial^{\varepsilon} I_{\sim}^{j}}\right|_{\Omega} \quad ; \quad j=1,2,3 \\
& { }_{C^{\varepsilon} j}=\left.\frac{\partial^{\varepsilon} \tilde{\alpha}^{2}}{\partial^{\varepsilon} I_{\sim}^{j}}\right|_{\underline{\Omega}} \quad ; \quad j=1,2,3 \\
& { }_{\sim}^{\varepsilon} d_{1}=\left.\frac{\partial^{\varepsilon} \tilde{\alpha}^{1}}{\partial \theta}\right|_{\underline{\Omega}} \\
& { }_{\sim}^{\varepsilon} d_{2}=\left.\frac{\partial^{\varepsilon} \tilde{\alpha}^{2}}{\partial \theta}\right|_{\underline{\Omega}} \\
& \left(\alpha_{t m}\right)_{\underline{\Omega}}=-\left.\frac{\partial^{\varepsilon} \tilde{\alpha}^{0}}{\partial \theta}\right|_{\underline{\Omega}}
\end{aligned}
$$


Substituting from (3.33) into (3.32)

$$
\begin{aligned}
{[\varepsilon]=} & \left.\varepsilon_{0}\right|_{\underline{\Omega}}[I]+{ }^{\varepsilon} b_{1}\left[\sigma^{[0]}\right]+{ }^{\varepsilon} b_{2}\left[\sigma^{[0]}\right]^{2}+ \\
& \sum_{j=1}^{3}{ }_{\sim}^{\varepsilon} a_{j}\left({ }_{\sim}^{\varepsilon} I^{j}[I]\right)+\sum_{j=1}^{3}{ }^{\varepsilon}{ }_{\sim} c_{1 j}\left({ }^{\varepsilon} I^{j}\left[\sigma^{[0]}\right]\right)+\sum_{j=1}^{3}{ }_{\sim}^{\varepsilon} c_{2 j}\left({ }_{\sim}^{\varepsilon} I_{\sim}^{j}\left[\sigma^{[0]}\right]^{2}\right)+ \\
& { }_{\sim} d_{1}\left(\theta-\theta_{\underline{\Omega}}\right)\left[\sigma^{[0]}\right]+{ }^{\varepsilon} \underline{\sim}_{2}\left(\theta-\theta_{\underline{\Omega}}\right)\left[\sigma^{[0]}\right]^{2}-\left(\alpha_{t m}\right)_{\underline{\Omega}}\left(\theta-\theta_{\underline{\Omega}}\right)[I]
\end{aligned}
$$

$\left.\varepsilon_{0}\right|_{\underline{\Omega}}$ is the initial strain in the known configuration $\underline{\Omega}$. This constitutive theory requires the determination of 14 material coefficients, ${ }^{\varepsilon} a_{j} ; j=1,2,3,{ }^{\varepsilon} \underline{b}_{i} ; i=1,2,{ }^{\varepsilon} c_{1 j},{ }^{\varepsilon}{ }_{2 j} ; j=1,2,3$, ${ }^{\varepsilon} \underline{d}_{j} ; j=1,2$ and $\alpha_{t m}$, all evaluated in a known configuration $\underline{\Omega}$. (3.34) is the most general constitutive theory for $[\varepsilon]$ as a function of $\left[\sigma^{[0]}\right]$ and $\theta$ resulting from the entropy inequality in Gibbs potential when we use either the approach given in section 3.1.1 or the approach in section 3.1.2. This theory is based on integrity and hence complete, but contains too many material coefficients to be determined experimentally or otherwise.

\section{Further simplifications}

The constitutive theory (3.34) requires the determination of too many material coefficients to be of practical use. If we only consider a constitutive theory for $[\varepsilon]$ that is linear in the components of $\left[\sigma^{[0]}\right]$ and further neglect the $\left(\theta-\theta_{\underline{\Omega}}\right)\left[\sigma^{[0]}\right]$ term, then (3.34) reduces to

$$
[\varepsilon]=\left.\varepsilon_{0}\right|_{\underline{\Omega}}[I]+{ }^{\varepsilon} \underline{b}_{1}\left[\sigma^{[0]}\right]+{ }^{\varepsilon} \underline{a}_{1} \operatorname{tr}\left[\sigma^{[0]}\right][I]+\left(\alpha_{t m}\right)_{\underline{\Omega}}\left(\theta-\theta_{\underline{\Omega}}\right)[I]
$$

This constitutive theory only requires three material coefficients, ${ }^{\varepsilon} \underline{a}_{1},{ }^{\varepsilon} \underline{b}_{1}$, and $\alpha_{t m}$ in a known configuration $\underline{\Omega}$.

\section{Remarks:}

1. It is perhaps meaningful to compare the constitutive theory (3.26) resulting from the Taylor series expansion of $\Psi$ and the constitutive theory (3.34) from the entropy 
inequality or from the theory of generators and invariants as the material coefficients in the two are now defined (in a known configuration $\underline{\Omega}$ ).

2. We observe that not all terms containing $\boldsymbol{\sigma}^{[0]}$ in the current configuration on the right hand side of (3.26) and (3.34) are the same.

3. Furthermore, the material coefficients in (3.26) are functions of $\left.\boldsymbol{\sigma}^{[0]}\right|_{\underline{\Omega}}$ and $\left.\theta\right|_{\underline{\Omega}}$, whereas the material coefficients in (3.34) are functions of the invariants of $\boldsymbol{\sigma}^{[0]}$ and $\theta$ in the known configuration $\underline{\Omega}$, hence in general the two sets of material coefficients are different.

4. Based on remarks (2) and (3) it is straightforward to conclude that the constitutive theories (3.26) and (3.34) are different. This raises the question regarding the superiority of one over the other. The constitutive theories in sections 3.1.1 and 3.1.2 are strictly based on the entropy inequality and integrity and hence are in precise agreement with the axioms and principles of continuum mechanics. The Taylor series expansion is based on axioms of smooth neighborhood, but it ignores the fundamental axiom that the coefficients in the constitutive theories must be functions of the combined invariants of the argument tensors.

5. Henceforth, in all further discussions and the determination of equivalence between the constitutive theories resulting from Gibbs potential and Helmholtz free energy density, we only consider the constitutive theory for $[\varepsilon]$ presented in sections 3.1.1 or 3.1.2 (as the two are identical in all aspects). 


\subsection{Constitutive theory for heat vector $q$}

The conditions resulting from the entropy inequality require that

$$
q_{i} g_{i} \leq 0
$$

be satisfied by the constitutive theories for $\boldsymbol{q}$ regardless of how they are derived. We can take two approaches to derive constitutive theory for $\boldsymbol{q}$. In the first approach, we strictly use (3.36) to derive constitutive theory for $\boldsymbol{q}$. Such constitutive theory for $\boldsymbol{q}$ will naturally satisfy the entropy inequality as it is derived using conditions resulting from it. In the second approach we determine the argument tensors of $\boldsymbol{q}$ and then use the theory of generators and invariants to derive the constitutive theories for $\boldsymbol{q}$. The constitutive theories for $\boldsymbol{q}$ derived using this approach must ensure that (3.36) is satisfied in order for the deforming matter to be in thermodynamic equilibrium during evolution. We present the derivation of the constitutive theories for $\boldsymbol{q}$ using both approaches and present comparisons of the resulting constitutive theories and make some remarks regarding their merits and shortcomings.

\subsubsection{Constitutive theory for $q$ using entropy inequality $[1,2,8]$}

This derivation based on (3.36) is fundamental and can be found in any textbook on continuum mechanics. We present details in the following to point out assumptions used in the derivation as it plays significant role when comparing this constitutive theory with the theories resulting from the theory of generators and invariants. Following references $[1,2,8]$, we begin with (3.36). Equation (3.36) implies

$$
\{q\}^{T}\{g\}=\beta \leq 0
$$


Using equality, we obtain

$$
\frac{\partial \beta}{\partial\{g\}}=\{q\} \quad \text { or } \quad \frac{\partial \beta}{\partial g_{i}}=q_{i}
$$

Hence

$$
\{q\}_{\boldsymbol{g}=0}=\left.\frac{\partial \beta}{\partial\{g\}}\right|_{\boldsymbol{g}=0}=0
$$

That is, heat flux vanishes in the absence of temperature gradient. Thus, the constitutive theory for $\boldsymbol{q}$ must be a function of $\boldsymbol{g}$. At this stage many possibilities exist, the simplest of course is assuming that $\boldsymbol{q}$ is proportional to $\boldsymbol{g}$, i.e. $\boldsymbol{q}$ is a linear function of $\boldsymbol{g}$.

$$
q_{i}(\boldsymbol{g})=-k_{i j}(\theta) g_{j} ; \quad \text { or } \quad\{q\}=-[k(\theta)]\{g\}
$$

from which we define

$$
\frac{\partial q_{i}}{\partial g_{j}}=-k_{i j}(\theta)
$$

Also, from (3.38)

$$
\frac{\partial^{2} \beta}{\partial g_{j} \partial g_{i}}=\frac{\partial q_{i}}{\partial g_{j}}=-k_{i j}(\theta) \leq 0
$$

From (3.42) we conclude that $[k]$ is positive semidefinite and all its three eigenvalues are non-negative. Equation (3.40) is Fourier heat conduction law. The thermal conductivity matrix $[k]$ does not have to be symmetric but is often assumed to be. This derivation is based on the assumption that $\boldsymbol{q}$ is a linear function of $\boldsymbol{g}$. In general, in this theory the coefficients of $[k]$ can be functions of temperature $\theta$.

\subsubsection{Constitutive theories for $q$ using theory of generators and invari- ants}

In this approach the heat vector $\boldsymbol{q}$, a tensor of rank one, is expressed as a linear combination of the combined generators (only tensors of rank one) of its argument tensors. The 
coefficients in the linear combination are assumed to be functions of the combined invariants of the argument tensors of $\boldsymbol{q}$ and temperature $\theta$. The material coefficients are derived by expanding each coefficient in the linear combination in Taylor series about a known configuration. In this approach it is obvious that the explicit form of the constitutive theory for $\boldsymbol{q}$ depends upon the argument tensors of $\boldsymbol{q}$ and the terms retained in the Taylor series expansion of the coefficients in the linear combination. We consider two possible obvious choices and present derivations of the resulting constitutive theories for $\boldsymbol{q}$ in the following.

\section{(a) Approach I}

In this derivation we assume that

$$
\boldsymbol{q}=\boldsymbol{q}(\boldsymbol{g}, \theta)
$$

$\boldsymbol{q}$ and $\boldsymbol{g}$ are tensors of rank one and $\theta$ is a tensor of rank zero. The only combined generator of rank one of the argument tensors $\boldsymbol{g}$ and $\theta$ is $\boldsymbol{g}$, hence based on the theory of generators and invariants $[1,2,8]$ we can write

$$
\boldsymbol{q}=-{ }^{q} \alpha \boldsymbol{g}
$$

The coefficient ${ }^{q} \alpha$ is a function of the combined invariants of $\boldsymbol{g}, \theta$, i.e. $\boldsymbol{g} \cdot \boldsymbol{g}$ and temperature $\theta$. Let us denote ${ }^{q} \underset{\sim}{I}=\boldsymbol{g} \cdot \boldsymbol{g}$ to simplify the details of further derivation. We note that (3.44) holds in the current configuration in which deformation is not known. Hence ${ }^{q} \alpha={ }^{q} \alpha\left({ }^{q} \underset{\sim}{I}, \theta\right)$ is not yet deterministic in (3.44). To determine material coefficients from (3.44), we expand ${ }^{q} \alpha\left({ }_{\sim}^{q}, \theta\right)$ in Taylor series about a known configuration $\underline{\Omega}$ in ${ }_{\sim}^{q} \underset{I}{\text { and }} \theta$ and retain only up to linear terms in ${ }^{q} \underset{\sim}{I}$ and $\theta$.

$$
{ }^{q} \alpha=\left.{ }^{q} \alpha\right|_{\underline{\Omega}}+\left.\frac{\partial^{q} \alpha}{\partial^{q} \underline{\underline{I}}}\right|_{\underline{\Omega}}\left({ }^{q} \underline{\sim}^{I}-\left.{ }^{q}\right|_{\underline{\Omega}}\right)+\left.\frac{\partial^{q} \alpha}{\partial \theta}\right|_{\underline{\Omega}}\left(\theta-\left.\theta\right|_{\underline{\Omega}}\right)
$$


Substituting from (3.45) into (3.44)

$$
\boldsymbol{q}=-\left(\left.{ }^{q} \alpha\right|_{\underline{\Omega}}+\left.\frac{\partial^{q} \alpha}{\partial^{q} \underline{\sim}}\right|_{\underline{\Omega}}\left({ }^{q} \underset{\sim}{I}-\left.{ }^{q} \underset{\sim}{I}\right|_{\underline{\Omega}}\right)+\left.\frac{\partial^{q} \alpha}{\partial \theta}\right|_{\underline{\Omega}}\left(\theta-\left.\theta\right|_{\underline{\Omega}}\right)\right) \boldsymbol{g}
$$

We note that $\left.{ }^{q} \alpha\right|_{\underline{\Omega}},\left.\frac{\partial^{q} \alpha}{\partial^{q} I}\right|_{\underline{\Omega}}$ and $\left.\frac{\partial^{q} \alpha}{\partial \theta}\right|_{\underline{\Omega}}$ are functions of $\left.{ }^{q} \underset{\sim}{I}\right|_{\underline{\Omega}}$ and $\left.\theta\right|_{\underline{\Omega}}$ whereas ${ }^{q} \alpha$ in (3.44) is a function of ${ }^{q} \underset{\sim}{I}, \theta$, both in the current configuration. From (3.46) we can write the following, noting that ${ }^{q} \underset{\sim}{I}=\boldsymbol{g} \cdot \boldsymbol{g}$.

$$
\boldsymbol{q}=-\left.{ }^{q} \alpha\right|_{\underline{\Omega}} \boldsymbol{g}-\left(\left.\frac{\partial^{q} \alpha}{\partial^{q} \underline{\sim}}\right|_{\underline{\Omega}}\right)(\boldsymbol{g} \cdot \boldsymbol{g}) \boldsymbol{g}+\left.\frac{\partial^{q} \alpha}{\partial^{q} \underline{\sim}}\right|_{\underline{\Omega}}(\boldsymbol{g} \cdot \boldsymbol{g})_{\underline{\Omega}} \boldsymbol{g}-\left.\frac{\partial^{q} \alpha}{\partial \theta}\right|_{\underline{\Omega}}\left(\theta-\theta_{\underline{\Omega}}\right) \boldsymbol{g}
$$

or

$$
\boldsymbol{q}=-\left(\left.{ }^{q} \alpha\right|_{\underline{\Omega}}-\left.\frac{\partial^{q} \alpha}{\partial^{q} \underline{\sim}}\right|_{\underline{\Omega}}(\boldsymbol{g} \cdot \boldsymbol{g})_{\underline{\Omega}}\right) \boldsymbol{g}-\left(\left.\frac{\partial^{q} \alpha}{\partial^{q} \underline{\underline{I}}}\right|_{\underline{\Omega}}\right)(\boldsymbol{g} \cdot \boldsymbol{g}) \boldsymbol{g}-\left.\frac{\partial^{q} \alpha}{\partial \theta}\right|_{\underline{\Omega}}\left(\theta-\theta_{\underline{\Omega}}\right) \boldsymbol{g}
$$

Let

$$
\begin{aligned}
& k\left(\theta_{\underline{\Omega}},\left.{ }^{q} \underset{\sim}{I}\right|_{\underline{\Omega}}\right)=\left.{ }^{q} \alpha\right|_{\underline{\Omega}}-\left.\frac{\partial^{q} \alpha}{\partial^{q} I}\right|_{\underline{\Omega}}(\boldsymbol{g} \cdot \boldsymbol{g})_{\underline{\Omega}} \\
& k_{1}\left(\theta_{\underline{\Omega}},\left.{ }^{q} \underset{\sim}{I}\right|_{\underline{\Omega}}\right)=\left.\frac{\partial^{q} \alpha}{\partial^{q} I}\right|_{\sim} \underline{\Omega} \\
& k_{2}\left(\theta_{\underline{\Omega}},\left.{ }^{q}{ }_{\sim}^{I}\right|_{\underline{\Omega}}\right)=\left.\frac{\partial^{q} \alpha}{\partial \theta}\right|_{\underline{\Omega}}
\end{aligned}
$$

Then

$$
\boldsymbol{q}=-k\left(\theta_{\underline{\Omega}},\left.{ }^{q}{ }_{\sim}^{I}\right|_{\underline{\Omega}}\right) \boldsymbol{g}-k_{1}\left(\theta_{\underline{\Omega}},\left.{ }^{q} \underset{\sim}{I}\right|_{\underline{\Omega}}\right)(\boldsymbol{g} \cdot \boldsymbol{g}) \boldsymbol{g}-k_{2}\left({ }_{\underline{\Omega}},\left.{ }^{q}{ }_{\sim}^{I}\right|_{\underline{\Omega}}\right)\left(\theta-\theta_{\underline{\Omega}}\right) \boldsymbol{g}
$$

This is the simplest possible constitutive theory for $\boldsymbol{q}$ based on the theory of generators and invariants. The only assumption in this theory is the truncation of the Taylor series in (3.45) beyond linear terms in ${ }^{q} \underset{\sim}{I}$ and $\theta$.

\section{(b) Approach II}

In this case, we consider

$$
\boldsymbol{q}=\boldsymbol{q}\left(\boldsymbol{\sigma}^{[0]}, \theta, \boldsymbol{g}\right)
$$


As shown in (2.17), this is a more general case due to dependence of $\boldsymbol{q}$ on $\boldsymbol{g}, \theta$ as well as $\boldsymbol{\sigma}^{[0]} \cdot \boldsymbol{q}$ is a tensor of rank one, whereas $\boldsymbol{\sigma}^{[0]}, \boldsymbol{g}$ and $\theta$ are symmetric tensor of rank two, tensor of rank one, and tensor of rank zero, respectively. Justification for retaining $\boldsymbol{\sigma}^{[0]}$ as an argument tensor of $\boldsymbol{q}$ will be discussed after we present details of the constitutive theory for $\boldsymbol{q}$ based on (3.51) by using the theory of generators and invariants. The combined generators of rank one of the argument tensors $\boldsymbol{\sigma}^{[0]}, \boldsymbol{g}$ and $\theta$ are

$$
\left\{{ }^{q} \underline{G}^{1}\right\}=\{g\} \quad ; \quad\left\{{ }^{q} G^{2}\right\}=\left[\sigma^{[0]}\right]\{g\} \quad ; \quad\left\{{ }^{q} G^{3}\right\}=\left[\sigma^{[0]}\right]^{2}\{g\}
$$

The combined invariants of the argument tensors $\boldsymbol{\sigma}^{[0]}, \boldsymbol{g}$ and $\theta$ are

$$
\begin{aligned}
& { }_{\sim}^{q} I^{1}=\operatorname{tr}\left(\left[\sigma^{[0]}\right]\right) \quad ; \quad{ }_{\sim}^{q} I^{2}=\operatorname{tr}\left(\left[\sigma^{[0]}\right]^{2}\right) \quad ; \quad{ }_{\sim}^{q} I^{3}=\operatorname{tr}\left(\left[\sigma^{[0]}\right]^{3}\right) \\
& { }_{\sim}^{q} I^{4}=\{g\} \cdot\{g\} \quad ; \quad{ }_{\sim}^{q} I^{5}=\{g\}^{T}\left[\sigma^{[0]}\right]\{g\} \quad ; \quad{ }_{\sim}^{q} I^{6}=\{g\}^{T}\left[\sigma^{[0]}\right]^{2}\{g\}
\end{aligned}
$$

We note that for $\left.{ }_{\sim}^{q}\right]^{j} ; j=1,2,3$ we could have also used $I_{\sigma[0]}, I_{\sigma^{[0]}}$ and $\mathbb{I I}_{\sigma[0]}$. As the two sets of invariants are related, the resulting constitutive theory remains unaffected. Using (3.52), we can write

$$
\boldsymbol{q}=-\sum_{i=1}^{3}{ }^{q} \alpha^{i}\left\{{ }^{q} G^{i}\right\}
$$

The coefficients ${ }^{q} \alpha^{i} ; i=1,2,3$ are functions of invariants ${ }_{\sim}^{q}{ }^{j} ; j=1,2, \ldots, 6$ and temperature $\theta$ in the current configuration. To determine the material coefficients from ${ }^{q} \alpha^{i} ; i=$ $1,2,3$ in (3.54), we consider Taylor series expansion of ${ }^{q} \alpha^{i} ; i=1,2,3$ in ${ }^{q} \underline{I}^{j} ; j=1,2, \ldots, 6$ and $\theta$ about a known configuration $\underline{\Omega}$ and retain only up to linear terms in $\theta$ and the invariants.

$$
{ }^{q} \alpha^{i}=\left.{ }^{q} \alpha^{i}\right|_{\underline{\Omega}}+\left.\sum_{j=1}^{6} \frac{\partial^{q} \alpha^{i}}{\partial \underline{\sim}^{q}{ }^{j}}\right|_{\underline{\Omega}}\left({ }_{\sim}^{q} I^{j}-\left({ }^{q} \underline{\sim}^{j}\right)_{\underline{\Omega}}\right)+\left.\frac{\partial^{q} \alpha^{i}}{\partial \theta}\right|_{\underline{\Omega}}\left(\theta-\theta_{\underline{\Omega}}\right) \quad ; \quad i=1,2,3
$$

$\left.{ }^{q} \alpha^{i}\right|_{\underline{\Omega}},\left.\frac{\partial^{q} \alpha^{i}}{\partial{ }_{\sim}^{q}{ }^{j}}\right|_{\underline{\Omega}} ; j=1,2, \ldots, 6$ and $\left.\frac{\partial^{q} \alpha^{i}}{\partial \theta}\right|_{\underline{\Omega}} ; i=1,2,3$ are functions of $\left.\theta\right|_{\underline{\Omega}},{ }_{\sim}^{q} I^{j} ; j=1,2, \ldots, 6$ whereas ${ }^{q} \alpha^{i}={ }^{q} \alpha^{i}\left(\left.\theta\right|_{\underline{\Omega}},\left.{ }_{\sim}^{q} \underline{\sim}^{j}\right|_{\underline{\Omega}} ; j=1,2, \ldots, 6, \theta, \underline{\sim}^{q} \underline{I}^{j} ; j=1,2, \ldots, 6\right)$. We substitute from 
(3.55) into (3.54).

$$
\boldsymbol{q}=-\sum_{i=1}^{3}\left(\left.{ }^{q} \alpha^{i}\right|_{\underline{\Omega}}+\sum_{j=1}^{6} \frac{\partial^{q} \alpha^{i}}{\partial \underline{I}^{q}{ }_{\underline{\Omega}}}{ }_{\underline{\Omega}}\left({ }^{q} I^{j}-\left(\stackrel{\sim}{q}^{q}\right)_{\underline{\Omega}}\right)+\left.\frac{\partial^{q} \alpha^{i}}{\partial \theta}\right|_{\underline{\Omega}}\left(\theta-\theta_{\underline{\Omega}}\right)\right)\left\{{ }^{q} \underline{G}^{i}\right\}
$$

Using (3.56), we collect coefficients (those defined in configuration $\Omega$ ) of $\left\{{ }^{q} G^{i}\right\},{ }^{q} I^{j}\left\{{ }^{q} G^{i}\right\}$, $\left(\theta-\theta_{\underline{\Omega}}\right)\left\{{ }^{q} G^{i}\right\} ; i=1,2,3$ and $j=1,2, \ldots, 6$ and define

$$
\begin{aligned}
& \left.{ }^{q} a_{i}=\left.{ }^{q} \alpha^{i}\right|_{\underline{\Omega}}-\left.\sum_{j=1}^{6} \frac{\partial^{q} \alpha^{i}}{\partial^{q} I^{j}}\right|_{\underline{\Omega}}{ }^{\left({ }^{q} I^{j}\right.}\right)_{\underline{\Omega}} \quad ; \quad i=1,2,3 \\
& { }^{q} b_{i j}=\left.\frac{\partial^{q} \alpha^{i}}{\partial{ }^{q j} j}\right|_{\underline{\Omega}} ; \quad i=1,2,3 \quad ; \quad j=1,2, \ldots, 6 \\
& { }^{q} c_{i}=\left.\frac{\partial^{q} \alpha^{i}}{\partial \theta}\right|_{\underline{\Omega}} ; i=1,2,3
\end{aligned}
$$

Using (3.57) in (3.56) we can write the following for $\boldsymbol{q}$.

$$
\boldsymbol{q}=-\sum_{i=1}^{3} a_{i} a_{i}\left\{{ }^{q} G^{i}\right\}-\sum_{i=1}^{3} \sum_{j=1}^{6}{ }^{q} b_{i j} \stackrel{q}{\sim}^{j}\left\{{ }^{q} G^{i}\right\}-\sum_{i=1}^{3} c_{i}^{q}\left(\theta-\theta_{\underline{\Omega}}\right)\left\{{ }^{q} G^{i}\right\}
$$

${ }^{q} a_{i},{ }^{q} b_{i j}$ and ${ }^{q} c_{i}$ are the material coefficients defined in the known configuration $\underline{\Omega}$. This constitutive theory for $\boldsymbol{q}$ uses full set of argument tensors and integrity and hence is complete. Unfortunately, it requires too many material coefficients (twenty four).

\section{Remarks:}

1. With some assumptions this constitutive theory for $\boldsymbol{q}$ can be simplified to yield an approximate constitutive theory in which the material coefficients may not be as many as in (3.58).This will undoubtedly limit the physics. If we limit the constitutive theory to be linear in the components of $\boldsymbol{\sigma}^{[0]}$, that is, we neglect generator $\left\{{ }^{q} G^{3}\right\}=\left[\sigma^{[0]}\right]^{2}\{g\}$ and invariants $\stackrel{q}{\sim} I^{2},{ }^{q} I^{3}$ and ${ }^{q} I^{6}$, the constitutive theory for $\boldsymbol{q}$ in 
(3.58) reduces to

$$
\begin{aligned}
\boldsymbol{q}= & -{ }^{q} a_{1}\{g\}-{ }^{q} a_{2}\left[\sigma^{[0]}\right]\{g\}-{ }^{q} b_{11}\left(\operatorname{tr}\left[\sigma^{[0]}\right]\right)\{g\}-{ }^{q} b_{14}(\{g\} \cdot\{g\})\{g\}- \\
& { }^{q} b_{15}\left(\{g\}^{T}\left[\sigma^{[0]}\right]\{g\}\right)\{g\}-{ }^{q} b_{21}\left(\operatorname{tr}\left[\sigma^{[0]}\right]\right)\left(\left[\sigma^{[0]}\right]\{g\}\right)- \\
& { }^{q} b_{24}(\{g\} \cdot\{g\})\left(\left[\sigma^{[0]}\right]\{g\}\right)-{ }^{q} b_{25}\left(\{g\}^{T}\left[\sigma^{[0]}\right]\{g\}\right)\left(\left[\sigma^{[0]}\right]\{g\}\right) \\
& -{ }^{q} c_{1}\left(\theta-\theta_{\underline{\Omega}}\right)\{g\}-{ }^{q} c_{2}\left(\theta-\theta_{\underline{\Omega}}\right)\left(\left[\sigma^{[0]}\right]\{g\}\right)
\end{aligned}
$$

This constitutive theory still requires ten material coefficients. If we further assume that the constitutive theory for $\boldsymbol{q}$ is linear in the components of $\left[\sigma^{[0]}\right]$, then the terms containing material coefficients ${ }^{q} b_{21}$ and ${ }^{q} b_{25}$ can be removed from (3.59).

$$
\begin{aligned}
\boldsymbol{q}= & -{ }^{q} a_{1}\{g\}-{ }^{q} a_{2}\left[\sigma^{[0]}\right]\{g\}-{ }^{q} b_{11}\left(\operatorname{tr}\left[\sigma^{[0]}\right]\right)\{g\}-{ }^{q} b_{14}(\{g\} \cdot\{g\})\{g\}- \\
& { }^{q} b_{15}\left(\{g\}^{T}\left[\sigma^{[0]}\right]\{g\}\right)\{g\}-{ }^{q} b_{24}(\{g\} \cdot\{g\})\left(\left[\sigma^{[0]}\right]\{g\}\right)- \\
& { }^{q} c_{1}\left(\theta-\theta_{\underline{\Omega}}\right)\{g\}-{ }^{q} c_{2}\left(\theta-\theta_{\underline{\Omega}}\right)\left(\left[\sigma^{[0]}\right]\{g\}\right)
\end{aligned}
$$

This constitutive theory requires eight material coefficients.

2. If we remove dependence of $\boldsymbol{q}$ on $\left[\sigma^{[0]}\right]$ in (3.60), then

$$
\boldsymbol{q}=-{ }^{q} a_{1}\{g\}-{ }^{q} b_{14}(\{g\} \cdot\{g\})\{g\}-{ }^{q} c_{1}\left(\theta-\theta_{\underline{\Omega}}\right)\{g\}
$$

This constitutive theory for $\boldsymbol{q}$ is the same as derived earlier (equation (3.50)). The coefficients in (3.61) are functions of $\left.\theta\right|_{\underline{\Omega}}$ and $(\boldsymbol{g} \cdot \boldsymbol{g})_{\underline{\Omega}}$.

3. To demonstrate the influence of stress field on heat conduction, we reduce the constitutive theory (3.60) to a most simplified possible theory by considering $\boldsymbol{q}$ to be a linear function of the components of $\{g\}$ as well as $\left[\sigma^{[0]}\right]$.

$$
\begin{gathered}
\boldsymbol{q}=-{ }^{q} a_{1}\{g\}-{ }^{q} a_{2}\left[\sigma^{[0]}\right]\{g\}-{ }^{q} b_{11}\left(\operatorname{tr}\left[\sigma^{[0]}\right]\right)\{g\}- \\
{ }^{q} c_{1}\left(\theta-\theta_{\underline{\Omega}}\right)\{g\}-{ }^{q} c_{2}\left(\theta-\theta_{\underline{\Omega}}\right)\left(\left[\sigma^{[0]}\right]\{g\}\right)
\end{gathered}
$$


If we neglect $\left(\theta-\theta_{\underline{\Omega}}\right)$ terms in (3.62), then we obtain

$$
\boldsymbol{q}=-{ }^{q} a_{1}\{g\}-{ }^{q} a_{2}\left[\sigma^{[0]}\right]\{g\}-{ }^{q} b_{11}\left(\operatorname{tr}\left[\sigma^{[0]}\right]\right)\{g\}
$$

The material coefficients in (3.63) are functions of $(\boldsymbol{g} \cdot \boldsymbol{g})_{\underline{\Omega}},\left(\operatorname{tr}\left[\sigma^{[0]}\right]\right)_{\underline{\Omega}},\left(\{g\}^{T}\left[\sigma^{[0]}\right]\{g\}\right)_{\underline{\Omega}}$ and $\theta_{\underline{\Omega}}$. This constitutive theory requires only three material coefficients. We can also write (3.63) as

$$
\boldsymbol{q}=-{ }^{q} a_{1}\{g\}-\left({ }^{q} a_{2}\left[\sigma^{[0]}\right]+{ }^{q} b_{11}\left(\operatorname{tr}\left[\sigma^{[0]}\right]\right)[I]\right)\{g\}
$$

If we let ${ }^{q} a_{1}=k,-{ }^{q} a_{2}=k_{1},{ }^{q} b_{11}=k_{2}$ where $k, k_{1}$ and $k_{2}$ are positive material coefficients, then (3.64) can be written as

$$
\boldsymbol{q}=-\left(k[I]-k_{1}\left[\sigma^{[0]}\right]-k_{2}\left(\operatorname{tr}\left[\sigma^{[0]}\right]\right)[I]\right)\{g\}=-[k]\{g\}
$$

in which $[k]$ is the effective conductivity matrix in the presence of stress field. The coefficient of $\{g\}$ in the second term on the right side of (3.64) is the influence of stress field on heat conduction (in the most simplified form of the constitutive theory for $\boldsymbol{q})$.

4. From (3.65) for 1 -D case (i.e. in $\mathbb{R}^{1}$ ) we can write

$$
q_{x 1}=-\left(k-\left(k_{1}+k_{2}\right) \sigma_{x 1 x 1}\right) \frac{\partial \theta}{\partial x_{1}}=-\underset{\sim}{k} \frac{\partial \theta}{\partial x_{1}}
$$

From (3.66) we clearly see that compression (negative $\sigma_{x 1 x 1}$ ) enhances heat conduction due to increased $\underset{\sim}{\mathrm{k}}$. This of course is due to faster vibrational energy transfer at the lower scale (mode of heat transfer) due to the reduced mean free path between the molecules because of compression. On the other hand, tension (positive $\sigma_{x 1 x 1}$ ) increases mean free path between the molecules, hence the vibrational energy transfer 
between the molecules is reduced compared to the unstressed state. In tension, effective $\underset{\sim}{k}$ is obviously reduced. We remark that influence of stress field on heat transfer is most significant under high compression or tension as it influences the mean free path significantly. We remark that all matters in reality are compressible, but the degree of compressibility may vary depending upon the matter and the application. 


\section{Chapter 4}

\section{Entropy inequality in terms of}

\section{Helmholtz free energy density $\Phi$ using}

conjugate pair $\boldsymbol{\sigma}^{[0]}$ and $\boldsymbol{\varepsilon}$

In this chapter we consider the entropy inequality expressed in terms of Helmholtz free energy density $\Phi$. If we consider $\boldsymbol{\sigma}^{[0]}$ and $\boldsymbol{\varepsilon}$ as conjugate stress and strain pair for finite deformation elasticity for isotropic, homogeneous solids, then we can derive the following for the entropy inequality in terms of $[1,2,8,23]$.

$$
\rho_{0}(\dot{\Phi}+\eta \dot{\theta})+\frac{|J| q_{i} g_{i}}{\theta}-\sigma_{k i}^{[0]} \dot{\varepsilon}_{i k} \leq 0
$$

Dot over quantities indicates material derivative. Based on (4.1), we can choose the following dependent variables in the constitutive theory and their argument tensors (for the most 
general case):

$$
\begin{aligned}
& \Phi=\Phi(\boldsymbol{\varepsilon}, \boldsymbol{g}, \theta) \\
& \eta=\eta(\boldsymbol{\varepsilon}, \boldsymbol{g}, \theta) \\
& \boldsymbol{\sigma}^{[0]}=\boldsymbol{\sigma}^{[0]}(\boldsymbol{\varepsilon}, \boldsymbol{g}, \theta) \\
& \boldsymbol{q}=\boldsymbol{q}(\boldsymbol{\varepsilon}, \boldsymbol{g}, \theta)
\end{aligned}
$$

Using the first equation in (4.2), we can determine $\dot{\Phi}$ using the chain rule of differentiation.

$$
\dot{\Phi}=\frac{\partial \Phi}{\partial \varepsilon_{i k}} \dot{\varepsilon}_{i k}+\frac{\partial \Phi}{\partial g_{i}} \dot{g}_{i}+\frac{\partial \Phi}{\partial \theta} \dot{\theta}
$$

Substituting $\dot{\Phi}$ from (4.3) into (4.1) and collecting coefficients of $\dot{\varepsilon}_{i k}$ and $\dot{\theta}$ we can write

$$
\left(\rho_{0} \frac{\partial \Phi}{\partial \varepsilon_{i k}}-\sigma_{k i}^{[0]}\right) \dot{\varepsilon}_{i k}+\rho_{0}\left(\frac{\partial \Phi}{\partial \theta}+\eta\right) \dot{\theta}+\frac{|J| q_{i} g_{i}}{\theta}+\frac{\partial \Phi}{\partial g_{i}} \dot{g}_{i} \leq 0
$$

Entropy inequality (4.4) must hold for all admissible choices of $\dot{\varepsilon}_{i k}, \dot{\theta}$ and $\dot{g}_{i}$. This is possible if the following conditions hold

$$
\begin{aligned}
& \rho_{0} \frac{\partial \Phi}{\partial \varepsilon_{i k}}-\sigma_{k i}^{[0]}=0 \\
& \frac{\partial \Phi}{\partial \theta}+\eta=0 \\
& \frac{\partial \Phi}{\partial g_{i}}=0 \\
& \text { and } \frac{|J| q_{i} g_{i}}{\theta} \leq 0 \quad \text { or } \quad q_{i} g_{i} \leq 0
\end{aligned}
$$

From (4.5) we conclude that $\eta$ is not a dependent variable in the constitutive theory as $\eta$ is deterministic from $-\frac{\partial \Phi}{\partial \theta}$ and $\boldsymbol{g}$ is not an argument tensor of $\Phi$ as $\frac{\partial \Phi}{\partial g_{i}}=0$ must hold. The remaining two conditions in (4.5) are

$$
\begin{aligned}
\sigma_{k i}^{[0]} & =\rho_{0} \frac{\partial \Phi}{\partial \varepsilon_{k i}} \\
q_{i} g_{i} & \leq 0
\end{aligned}
$$


Thus, constitutive theory for $\boldsymbol{\sigma}^{[0]}$ is deterministic using (4.6) and the constitutive theory for $\boldsymbol{q}$ must satisfy (4.7). Equations (4.6) and (4.7) are two fundamental relations resulting from the entropy inequality expressed in terms of $\Phi$ that allow determination of constitutive theory for $\boldsymbol{\sigma}^{[0]}$ and $\boldsymbol{q}$. Based on (4.5), (4.2) reduces to

$$
\begin{aligned}
& \Phi=\Phi(\boldsymbol{\varepsilon}, \theta) \\
& \boldsymbol{\sigma}^{[0]}=\boldsymbol{\sigma}^{[0]}(\boldsymbol{\varepsilon}, \theta) \\
& \boldsymbol{q}=\boldsymbol{q}(\boldsymbol{\varepsilon}, \theta, \boldsymbol{g})
\end{aligned}
$$

Expressions in (4.8) are also of fundamental importance in the derivation of the constitutive theories. We note that using Helmholtz free energy density, the constitutive theory results in $\boldsymbol{\sigma}^{[0]}$ as a function of $\boldsymbol{\varepsilon}$ and $\theta$ (from equation (4.6)) and the argument tensors of $\boldsymbol{q}$ could possibly be $\boldsymbol{\varepsilon}, \theta$, and $\boldsymbol{g}$. However, when the entropy inequality is expressed in terms of Gibbs potential (chapter 3) the constitutive theory expresses $\boldsymbol{\varepsilon}$ as a function of $\boldsymbol{\sigma}^{[0]}$ and $\theta$ and the possible argument tensors of $\boldsymbol{q}$ in this case are $\boldsymbol{\sigma}^{[0]}, \boldsymbol{g}$ and $\theta$. 


\section{Chapter 5}

\section{Constitutive theory for stress tensor $\boldsymbol{\sigma}^{[0]}$}

\section{and heat vector $q$ using Helmholtz free}

\section{energy density}

In this chapter we present basic steps of the derivations and compact summaries of the constitutive theories for $\boldsymbol{\sigma}^{[0]}$ and $\boldsymbol{q}$ that are possible using entropy inequailty expressed in terms of Helmholtz free energy density. Details of the derivations can be found in $[1,23]$.

\subsection{Constitutive theory for stress tensor $\boldsymbol{\sigma}^{[0]}$}

The constitutive theory for $\boldsymbol{\sigma}^{[0]}$ can be derived using (4.6) and (4.8). As in section 3 , here also there are three possible approaches: (i) assuming $\Phi$ to be a function of the invariants of $\boldsymbol{\varepsilon}$ and $\theta$ and using (4.6), (ii) using the first equation in (4.2) in conjunction with the theory of generators and invariants [1-22] and (iii) using Taylor series expansion of $\Phi$ 
in $\boldsymbol{\varepsilon}$ about a known configuration and then using (4.6). Here also we examine the resulting constitutive theories from these three approaches to determine the conditions under which there is equivalence between the resulting three forms of the constitutive theories.

\subsubsection{Constitutive theory for $\boldsymbol{\sigma}^{[0]}$ using (4.6) and assuming $\Phi$ to be a function of the invariants of $\varepsilon[1,2,8,23]$}

Consider

$$
\Phi=\Phi\left(I_{\varepsilon}, I_{\varepsilon}, \mathbb{I I}_{\varepsilon}, \theta\right)
$$

Following the procedure and details presented in section 3.1.1 (equations (3.4) - (3.15)), it is straightforward to derive the following

$$
\left[\sigma^{[0]}\right]={ }^{\sigma} \alpha^{0}[I]+{ }^{\sigma} \alpha^{1}[\varepsilon]+{ }^{\sigma} \alpha^{2}[\varepsilon]^{2}
$$

in which the coefficients ${ }^{\sigma} \alpha^{i} ; i=0,1,2$ are functions of the invariants of $\boldsymbol{\varepsilon}$ and $\theta$ in the current configuration. As in (3.15), this theory in the present form is not usable due to the fact that ${ }^{\sigma} \alpha^{i} ; i=0,1,2$ are functions of unknown deformations in the current configuration.

However, (5.2) is the fundamental form of the constitutive theory for $\boldsymbol{\sigma}^{[0]}$ in this approach.

\subsubsection{Constitutive theory for $\boldsymbol{\sigma}^{[0]}$ using (4.8) and the theory of genera- tors and invariants [1-23]}

Consider

$$
\boldsymbol{\sigma}^{[0]}=\boldsymbol{\sigma}^{[0]}(\boldsymbol{\varepsilon}, \theta)
$$


Following the procedure and details given in 3.1.2 (equations (3.16) - (3.18)), we can easily derive the following

$$
\left[\sigma^{[0]}\right]={ }^{\sigma} \alpha^{0}[I]+{ }^{\sigma} \alpha^{1}[\varepsilon]+{ }^{\sigma} \alpha^{2}[\varepsilon]^{2}
$$

in which the coefficients ${ }^{\sigma} \alpha^{i} ; i=0,1,2$ are functions of the invariants of $\boldsymbol{\varepsilon}$ and $\theta$ in the current configuration. (5.4) is the same as (5.2). Remarks similar to those in section 3.1.2 apply here as well.

\subsubsection{Constitutive theory for $\boldsymbol{\sigma}^{[0]}$ by expanding $\Phi$ in Taylor series in $\varepsilon$ about a known configuration and then using (4.6) $[1,2,8,23]$}

We consider $\Phi=\Phi(\boldsymbol{\varepsilon}, \theta)$ and expand $\Phi$ in $\boldsymbol{\varepsilon}$ using Taylor series about a known configuration $\underline{\Omega}$. The derivation follows exactly the same steps as in section 3.1 .3 (employing similar assumptions) and we obtain

$$
\sigma_{m n}^{[0]}=\left.\sigma_{m n}^{0}\right|_{\underline{\Omega}}+\left.\overline{\bar{C}}_{m n i j}\right|_{\underline{\Omega}} \varepsilon_{i j}+\left.\left(\underline{\bar{C}}_{m n i j k l}\right)\right|_{\underline{\Omega}} \varepsilon_{i j} \varepsilon_{k l}+\ldots
$$

\section{Remarks:}

The following remarks are similar to those at the end of section 3.1.3 but are presented here for completeness.

1. We note that (5.5) and (5.4), (5.2) are similar in the sense that all these three forms contain exactly the same tensors on the left and right side of the equality that are in the current configuration.

2. In (5.2) and (5.4) the coefficients ${ }^{\sigma} \alpha^{i}$ are defined in the current configuration, whereas in $(5.5)$ the coefficients are in the known configuration $\underline{\Omega}$. Hence, constitutive theory 
(5.5) is quite different compared to (5.2) or (5.4).

3. Based on the derivations given in sections 3.1.1 and 3.1.2, it is clear that the Taylor series expansion in $\Phi=\Phi(\boldsymbol{\varepsilon}, \theta)$ must be limited up to the cubic terms in $\boldsymbol{\varepsilon}$. Inclusion of further higher degree terms in $\boldsymbol{\varepsilon}$ is non-physical and it is not supported by the derivations that are strictly based on the entropy inequality.

4. From Taylor series expansion it is clear that the coefficients in (5.5) are functions of $\boldsymbol{\varepsilon}$ and $\theta$ in the known configuration $\underline{\Omega}$, whereas the coefficients ${ }^{\sigma} \alpha^{i} ; i=0,1,2$ in (5.2) and (5.4) are functions of invariants of $\boldsymbol{\varepsilon}$ in the current configuration.

5. The issue of whether (5.5) is superior over (5.2) or (5.4) can only be addressed after we determine the material coefficients using ${ }^{\sigma} \alpha^{i} ; i=0,1,2$. We present details in the following section.

6. For homogeneous isotropic solid matter the coefficients in (5.5) can be simplified [1].

\subsubsection{Determination of material coefficients using ${ }^{\sigma} \alpha^{i} ; i=0,1,2$ in} $(5.2)$ or $(5.4)[\mathbf{1}, \mathbf{2}, \mathbf{8}, \mathbf{2 3}]$

Consider

$$
\boldsymbol{\sigma}^{[0]}={ }^{\sigma} \alpha^{0}[I]+{ }^{\sigma} \alpha^{1}[\varepsilon]+{ }^{\sigma} \alpha^{2}[\varepsilon]^{2}
$$

in which

$$
{ }^{\sigma} \alpha^{i}={ }^{\sigma} \alpha^{i}\left(I_{\varepsilon}, I_{\varepsilon}, I I I_{\varepsilon}, \theta\right)
$$

If we let ${ }^{\sigma} I^{1}=I_{\varepsilon},{ }^{\sigma} I^{2}=I_{\varepsilon}$ and ${ }^{\sigma} I^{3}=\mathbb{I I}_{\varepsilon}$ and follow the derivation in section 3.1.4 and define the coefficients as in (5.8), then we can obtain (5.9) as a constitutive theory for 
second Piola-Kirchoff stress $\boldsymbol{\sigma}^{[0]}$.

$$
\begin{aligned}
& \left.\sigma_{0}\right|_{\underline{\Omega}}=\left.{ }^{\sigma} \alpha^{0}\right|_{\underline{\Omega}}-\left.\sum_{j=1}^{3} \frac{\partial^{\sigma} \alpha^{0}}{\partial{ }^{\sigma}{ }^{j}}\right|_{\underline{\Omega}}\left({ }^{\sigma} I^{j}\right)_{\underline{\Omega}} \\
& \sigma_{\tilde{a}_{j}}=\left.\frac{\partial^{\sigma} \alpha^{0}}{\partial{ }_{\sim}^{\sigma j}}\right|_{\underline{\Omega}} \quad ; \quad j=1,2,3 \\
& \tilde{\sigma}_{i}=\left.{ }^{\sigma} \alpha^{i}\right|_{\underline{\Omega}}-\left.\sum_{j=1}^{3} \frac{\partial^{\sigma} \alpha^{i}}{\partial{ }^{\sigma} I^{j}}\right|_{\underline{\Omega}} \quad ; \quad i=1,2 \\
& { }^{\sigma} \tilde{c}_{1 j}=\left.\frac{\partial^{\sigma} \alpha^{1}}{\partial^{\sigma}{ }^{j}{ }^{j}}\right|_{\underline{\Omega}} \quad ; \quad j=1,2,3 \\
& \sigma_{\tilde{C}_{2 j}}=\left.\frac{\partial^{\sigma} \alpha^{2}}{\partial{ }_{\sim}^{\sigma} I^{j}}\right|_{\underline{\Omega}} \quad ; \quad j=1,2,3 \\
& \sigma \tilde{d}_{1}=\left.\frac{\partial{ }^{\sigma} \alpha^{1}}{\partial \theta}\right|_{\underline{\Omega}} \\
& { }^{\sigma} \tilde{d}_{2}=\left.\frac{\partial^{\sigma} \alpha^{2}}{\partial \theta}\right|_{\underline{\Omega}} \\
& \left(\tilde{\alpha}_{t m}\right)_{\underline{\Omega}}=-\left.\frac{\partial^{\sigma} \alpha^{0}}{\partial \theta}\right|_{\underline{\Omega}} \\
& {\left[\sigma^{[0]}\right]=\left.\sigma_{0}\right|_{\underline{\Omega}}[I]+\tilde{b}_{1}[\varepsilon]+\tilde{b}_{2}[\varepsilon]^{2}+} \\
& \sum_{j=1}^{3}{ }^{\sigma} \tilde{a}_{j}\left({ }_{\sim}^{\sigma}{ }_{\sim}^{j}[I]\right)+\sum_{j=1}^{3}{ }^{\sigma} \tilde{c}_{1 j}\left({ }_{\sim}^{\sigma} I^{j}[\varepsilon]\right)+\sum_{j=1}^{3}{ }^{\sigma} \tilde{c}_{2 j}\left({ }_{\sim}^{\sigma} I^{j}[\varepsilon]^{2}\right)+ \\
& \sigma \tilde{d}_{1}\left(\theta-\theta_{\underline{\Omega}}\right)[\varepsilon]+{ }^{\sigma} \tilde{d}_{2}\left(\theta-\theta_{\underline{\Omega}}\right)[\varepsilon]^{2}-\left(\tilde{\alpha}_{t m}\right)_{\underline{\Omega}}\left(\theta-\theta_{\underline{\Omega}}\right)[I]
\end{aligned}
$$

$\left.\sigma_{0}\right|_{\underline{\Omega}}$ is the initial stress in the known configuration $\underline{\Omega}$. This constitutive theory requires the determination of 14 material coefficients, ${ }^{\sigma} \tilde{a}_{j} ; j=1,2,3, \tilde{\sigma}_{i} ; i=1,2,{ }^{\sigma} \tilde{c}_{1 j},{ }^{\sigma} \tilde{c}_{2 j} ; j=$ $1,2,3,{ }^{\sigma} \tilde{d}_{j} ; j=1,2$ and $\tilde{\alpha}_{t m}$, all evaluated in a known configuration $\underline{\Omega}$. (5.9) is the general constitutive theory for $\left[\sigma^{[0]}\right]$ as a function of $[\varepsilon]$ resulting from the entropy inequality in Helmholtz free energy density $\Phi$ when we use either the approach given in section 5.1.1 or the approach in section 5.1.2. 


\section{Further simplifications}

The constitutive theory (5.9) requires the determination of many material coefficients.

If we only consider a constitutive theory for $\left[\sigma^{[0]}\right]$ that is linear in the components of $[\varepsilon]$ and further neglect the $\left(\theta-\theta_{\underline{\Omega}}\right)[\varepsilon]$ term, then (5.9) reduces to

$$
\left[\sigma^{[0]}\right]=\left.\sigma_{0}\right|_{\underline{\Omega}}[I]+{ }^{\sigma} \tilde{b}_{1}[\varepsilon]+{ }^{\sigma} \tilde{a}_{1} \operatorname{tr}[\varepsilon][I]+\left(\tilde{\alpha}_{t m}\right)_{\underline{\Omega}}\left(\theta-\theta_{\underline{\Omega}}\right)[I]
$$

This constitutive theory only requires three material coefficients, ${ }^{\sigma} \tilde{a}_{1},{ }^{\sigma} \tilde{b}_{1}$, and $\tilde{\alpha}_{t m}$ in a known configuration $\underline{\Omega}$.

\section{Remarks:}

1. It is perhaps meaningful to compare the constitutive theory (5.5) resulting from the Taylor series expansion and the constitutive theory (5.9) resulting from the entropy inequality or from the theory of generators and invariants as the material coefficients in the two are now defined (in a known configuration $\underline{\Omega}$ ).

2. We observe that not all terms involving $\boldsymbol{\varepsilon}$ in the current configuration on the right hand side of (5.5) and (5.9) are the same.

3. Furthermore, the material coefficients in (5.5) are functions of $\left.\boldsymbol{\varepsilon}\right|_{\underline{\Omega}}$ and $\left.\theta\right|_{\underline{\Omega}}$. The material coefficients in (5.9) are functions of the invariants of $\boldsymbol{\varepsilon}$ and $\theta$ in the known configuration $\underline{\Omega}$, hence in general the two sets of material coefficients are different.

4. Based on remarks (2) and (3) it is straightforward to conclude that the constitutive theories (5.5) and (5.9) are not the same. This raises a question regarding the superiority of one over the other. The constitutive theories in sections 5.1.1 and 5.1.2 are 
strictly based on the entropy inequality and integrity and hence are in precise agreement with the axioms and principles of continuum mechanics. The Taylor series expansion, though based on axioms of smooth neighborhood, ignores the fundamental axiom that the coefficients must be functions of the combined invariants of the argument tensors.

5. Henceforth, in all further discussions and the determination of equivalence between the constitutive theories resulting from Gibbs potential and Helmholtz free energy density, we only consider the constitutive theory for $\left[\sigma^{[0]}\right]$ presented in sections 5.1.1 or 5.1.2 (as the two are identical in all aspects).

\subsection{Constitutive theory for heat vector $q$}

The conditions resulting from the entropy inequality require that

$$
q_{i} g_{i} \leq 0
$$

be satisfied by the constitutive theory for $\boldsymbol{q}$ regardless of how it is derived. This condition is the same as in section 3.2, hence the constitutive theory for $\boldsymbol{q}$ derived in section 3.2.1 hold here as well. In the following, we only present the final forms of the constitutive theories for $\boldsymbol{q}$.

\subsubsection{Constitutive theory for $q$ using entropy inequality $[1,2,8]$}

Beginning with (5.11) and following the derivation in section 3.2.1, we obtain exactly the same constitutive theory for $\boldsymbol{q}$ as in section 3.2.1.

$$
\boldsymbol{q}(\boldsymbol{g})=-k_{i j}(\theta) \boldsymbol{g} \quad \text { or } \quad\{q\}=-[k(\theta)]\{g\}
$$




\subsubsection{Constitutive theory for $q$ using the theory of generators and in- variants $[1,2,8,23]$}

As in section 3.2.2, here also we can consider two approaches

\section{(a) Approach I}

Consider

$$
\boldsymbol{q}=\boldsymbol{q}(\boldsymbol{g}, \theta)
$$

which is the same as (3.43), hence would result in the following constitutive theory for $\boldsymbol{q}$ (see section 3.2.2, Approach I).

$$
\boldsymbol{q}=-k\left(\theta_{\underline{\Omega}},\left.{ }^{q} \underset{\sim}{I}\right|_{\underline{\Omega}}\right) \boldsymbol{g}-k_{1}\left(\theta_{\underline{\Omega}},\left.{ }^{q} \underset{\sim}{I}\right|_{\underline{\Omega}}\right)(\boldsymbol{g} \cdot \boldsymbol{g}) \boldsymbol{g}-k_{2}\left(\theta_{\underline{\Omega}},{ }^{q} \underline{\underline{\Omega}}_{\underline{\Omega}}\right)\left(\theta-\theta_{\underline{\Omega}}\right) \boldsymbol{g}
$$

The constitutive theory for $\boldsymbol{q}$ is identical to (3.50).

\section{(b) Approach II}

Consider

$$
\boldsymbol{q}=\boldsymbol{q}(\boldsymbol{\varepsilon}, \boldsymbol{g}, \theta)
$$

and define

$$
\left\{{ }^{q} \tilde{G}^{1}\right\}=\{g\} \quad ; \quad\left\{{ }^{q} \tilde{G}^{2}\right\}=[\varepsilon]\{g\} \quad ; \quad\left\{{ }^{q} \tilde{G}^{3}\right\}=\{g\}^{T}[\varepsilon]\{g\}
$$

Then using the theory of generators and invariants we can write

$$
\boldsymbol{q}=-\sum_{i=1}^{3} \tilde{\alpha}^{i}\left\{{ }^{q} \tilde{G}^{i}\right\}
$$


Following the derivation in section 3.2.2, Approach II, we can obtain the constitutive theory for $\boldsymbol{q}$ as in (5.19). The coefficients in (5.19) are defined in (5.18).

$$
\begin{aligned}
& { }^{q} \tilde{a}_{i}=\left.{ }^{q} \alpha^{i}\right|_{\underline{\Omega}}-\left.\sum_{j=1}^{6} \frac{\partial^{q} \tilde{\alpha}^{i}}{\partial^{q} \tilde{I}^{j}}\right|_{\underline{\Omega}}\left({ }^{q} \tilde{I}^{j}\right)_{\underline{\Omega}} \quad ; \quad i=1,2,3 \\
& { }^{q} \tilde{b}_{i j}=\left.\frac{\partial^{q} \tilde{\alpha}^{i}}{\partial \tilde{I}^{j}}\right|_{\underline{\Omega}} ; \quad i=1,2,3 \quad ; \quad j=1,2, \ldots, 6 \\
& { }^{q} \tilde{c}_{i}=\left.\frac{\partial^{q} \tilde{\alpha}^{i}}{\partial \theta}\right|_{\underline{\Omega}} ; \quad i=1,2,3 \\
& \boldsymbol{q}=-\sum_{i=1}^{3}{ }^{q} \tilde{a}_{i}\left\{{ }^{q} \tilde{G}^{i}\right\}+\sum_{i=1}^{3} \sum_{j=1}^{6} \tilde{b}_{i j}{ }^{q} \tilde{I}^{j}\left\{{ }^{q} \tilde{G}^{i}\right\}-\sum_{i=1}^{3} q \tilde{c}_{i}\left(\theta-\theta_{\underline{\Omega}}\right)\left\{{ }^{q} \tilde{G}^{i}\right\}
\end{aligned}
$$

where ${ }^{q} \tilde{I}^{j} ; j=1,2, \ldots, 6$ are combined invariants of the argument tensors of $\boldsymbol{q}$ in (5.15). ${ }^{q} \tilde{a}_{i},{ }^{q} \tilde{b}_{i j}$ and ${ }^{q} \tilde{c}_{i}$ are the material coefficients defined in the known configuration $\underline{\Omega}$. This constitutive theory for $\boldsymbol{q}$ uses integrity and hence is complete. Unfortunately, it requires too many material coefficients (twenty four).

\section{Remarks:}

1. With some assumptions this constitutive theory for $\boldsymbol{q}$ can be simplified to yield an approximate constitutive theory in which the material coefficients may not be as many as in (5.19).This will undoubtedly limit the physics. If we limit the constitutive theory to be linear in the components of $\boldsymbol{\varepsilon}$, that is, we neglect generator $\left\{{ }^{q} \tilde{G}^{3}\right\}=[\varepsilon]^{2}\{g\}$ and invariants ${ }^{q} \tilde{I}^{2},{ }^{q} \tilde{I}^{3}$ and ${ }^{q} \tilde{I}^{6}$, (similar to those in (3.53)) the constitutive theory for $\boldsymbol{q}$ in (5.19) now reduces to

$$
\begin{aligned}
\boldsymbol{q}= & -{ }^{q} \tilde{a}_{1}\{g\}-{ }^{q} \tilde{a}_{2}[\varepsilon]\{g\}-{ }^{q} \tilde{b}_{11}(\operatorname{tr}[\varepsilon])\{g\}-{ }^{q} \tilde{b}_{14}(\{g\} \cdot\{g\})\{g\}- \\
& { }^{q} \tilde{b}_{15}\left(\{g\}^{T}[\varepsilon]\{g\}\right)\{g\}-{ }^{q} \tilde{b}_{21}(\operatorname{tr}[\varepsilon])\left(\left[\sigma^{[0]}\right]\{g\}\right)- \\
& { }^{q} \tilde{b}_{24}(\{g\} \cdot\{g\})([\varepsilon]\{g\})-{ }^{q} \tilde{b}_{25}\left(\{g\}^{T}[\varepsilon]\{g\}\right)([\varepsilon]\{g\})- \\
& { }^{q} \tilde{c}_{1}\left(\theta-\theta_{\underline{\Omega}}\right)\{g\}-{ }^{q} \tilde{c}_{2}\left(\theta-\theta_{\underline{\Omega}}\right)([\varepsilon]\{g\})
\end{aligned}
$$


This constitutive theory still requires ten material coefficients. If we further assume that the constitutive theory for $\boldsymbol{q}$ is linear in the components of $\boldsymbol{\varepsilon}$, then the terms containing material coefficients ${ }^{q} \tilde{b}_{21}$ and ${ }^{q} \tilde{b}_{25}$ can be removed from (5.20).

$$
\begin{aligned}
\boldsymbol{q}= & -{ }^{q} \tilde{a}_{1}\{g\}-{ }^{q} \tilde{a}_{2}[\varepsilon]\{g\}-{ }^{q} \tilde{b}_{11}(\operatorname{tr}[\varepsilon])\{g\}-{ }^{q} \tilde{b}_{14}(\{g\} \cdot\{g\})\{g\}- \\
& { }^{q} \tilde{b}_{15}\left(\{g\}{ }^{T}[\varepsilon]\{g\}\right)\{g\}-{ }^{q} \tilde{b}_{24}(\{g\} \cdot\{g\})([\varepsilon]\{g\})- \\
& { }^{q} \tilde{C}_{1}\left(\theta-\theta_{\underline{\Omega}}\right)\{g\}-{ }^{q} \tilde{C}_{2}\left(\theta-\theta_{\underline{\Omega}}\right)([\varepsilon]\{g\})
\end{aligned}
$$

This constitutive theory requires eight material coefficients.

2. If we remove dependence of $\boldsymbol{q}$ on $[\varepsilon]$ in (5.21), then

$$
\boldsymbol{q}=-{ }^{q} \tilde{a}_{1}\{g\}-{ }^{q} \tilde{b}_{14}(\{g\} \cdot\{g\})\{g\}-{ }^{q} \tilde{c}_{1}\left(\theta-\theta_{\underline{\Omega}}\right)\{g\}
$$

This constitutive theory for $\boldsymbol{q}$ is the same as derived earlier (equation (3.50)). The coefficients in (5.22) are functions of $\left.\theta\right|_{\underline{\Omega}}$ and $(\boldsymbol{g} \cdot \boldsymbol{g})_{\underline{\Omega}}$.

3. To demonstrate the significance of strain field on heat conduction, we reduce the constitutive theory (5.21) to a most simplified possible theory by considering $\boldsymbol{q}$ to be a linear function of the components of $\{g\}$ as well as $[\varepsilon]$.

$$
\begin{gathered}
\boldsymbol{q}=-{ }^{q} \tilde{a}_{1}\{g\}-{ }^{q} \tilde{a}_{2}[\varepsilon]\{g\}-{ }^{q} \tilde{b}_{11}(\operatorname{tr}[\varepsilon])\{g\}- \\
{ }^{q} \tilde{c}_{1}\left(\theta-\theta_{\underline{\Omega}}\right)\{g\}-{ }^{q} \tilde{c}_{2}\left(\theta-\theta_{\underline{\Omega}}\right)([\varepsilon]\{g\})
\end{gathered}
$$

If we neglect $\left(\theta-\theta_{\underline{\Omega}}\right)$ terms in (5.23) as done routinely, we obtain

$$
\boldsymbol{q}=-{ }^{q} \tilde{a}_{1}\{g\}-{ }^{q} \tilde{a}_{2}[\varepsilon]\{g\}-{ }^{q} \tilde{b}_{11}(\operatorname{tr}[\varepsilon])\{g\}
$$

The material coefficients in (5.24) are functions of $(\boldsymbol{g} \cdot \boldsymbol{g})_{\underline{\Omega}},(\operatorname{tr}[\varepsilon])_{\underline{\Omega}},\left(\{g\}^{T}[\varepsilon]\{g\}\right)_{\underline{\Omega}}$. This constitutive theory requires only three material coefficients. We can also write (5.24) as

$$
\boldsymbol{q}=-{ }^{q} \tilde{a}_{1}\{g\}-\left({ }^{q} \tilde{a}_{2}[\varepsilon]+{ }^{q} \tilde{b}_{11}(\operatorname{tr}[\varepsilon])[I]\right)\{g\}
$$


If we let ${ }^{q} \tilde{a}_{1}=\bar{k},-{ }^{q} \tilde{a}_{2}=\bar{k}_{1}$ and $-{ }^{q} \tilde{b}_{11}=\bar{k}_{2}$ where $\bar{k}, \bar{k}_{1}$ and $\bar{k}_{2}$ are positive material coefficients, then (5.25) can be written as

$$
q=-\left(\bar{k}[I]-\bar{k}_{1}[\varepsilon]-\bar{k}_{2}(\operatorname{tr}[\varepsilon])[I]\right)\{g\}=-[\bar{k}]\{g\}
$$

in which $[\bar{k}]$ is the effective conductivity matrix in the presence of strain field. The coefficient of $\{g\}$ in the second term on the right side of (5.25) is the influence of strain field on heat conduction (in the most simplified form of the constitutive theory for $\boldsymbol{q}$ ). From (5.26) for 1-D case (i.e. in $\mathbb{R}^{1}$ ) we can write

$$
q_{x 1}=-\left(\bar{k}-\left(\bar{k}_{1}+\bar{k}_{2}\right) \varepsilon_{x 1 x 1}\right) \frac{\partial \theta}{\partial x_{1}}=-\bar{c} \frac{\partial \theta}{\partial x_{1}}
$$

This is similar to (3.66), hence the comments made in Remark 4 (following equation (3.66)) hold here as well but are not repeated for the sake of brevity. 


\title{
Chapter 6
}

\section{Comparison of the constitutive theories}

\author{
resulting from the entropy inequality
}

\section{expressed in terms of Gibbs potential $\Psi$}

\section{and Helmholtz free energy density $\Phi$}

In this chapter we compare the constitutive theories derived using Gibbs potential in chapter 3 with the constitutive theories derived using Helmholtz free energy density $\Phi$ in chapters 4 and 5 to determine when there is equivalence between these theories. We use the notation $G$ to indicate Gibbs potential and $H$ for Helmholtz free energy density. 


\subsection{Constitutive theories for $\sigma^{[0]}$ and $\varepsilon$}

\subsubsection{Basic forms resulting from the entropy inequality}

The most basic forms resulting from the entropy inequality or the theory of generators and invariants are equations (3.15) or (3.17) and (5.2) or (5.4).

$$
\begin{array}{lll}
{[\varepsilon]={ }^{\varepsilon} \tilde{\alpha}^{0}[I]+{ }^{\varepsilon} \tilde{\alpha}^{1}\left[\sigma^{[0]}\right]+{ }^{\varepsilon} \tilde{\alpha}^{2}\left[\sigma^{[0]}\right]^{2}} & : & G \\
{\left[\sigma^{[0]}\right]={ }^{\sigma} \tilde{\alpha}^{0}[I]+{ }^{\sigma} \tilde{\alpha}^{1}[\varepsilon]+{ }^{\sigma} \tilde{\alpha}^{2}[\varepsilon]^{2}} & : & H
\end{array}
$$

in which

$$
\begin{aligned}
& \varepsilon \tilde{\alpha}^{i}={ }^{\varepsilon} \tilde{\alpha}^{i}\left(I_{\sigma[0]}, I_{\sigma[0]}, I I_{\sigma^{[0]}}, \theta\right) \\
& { }^{\sigma} \tilde{\alpha}^{i}={ }^{\sigma} \tilde{\alpha}^{i}\left(I_{\varepsilon}, I_{\varepsilon}, I I_{\varepsilon}, \theta\right) \quad ; \quad i=0,1,2
\end{aligned}
$$

It is rather obvious that there is no equivalence between these two theories, i.e. $\boldsymbol{\sigma}^{[0]}$ if determined from (6.1) is not the same as $\boldsymbol{\sigma}^{[0]}$ in (6.2). Likewise, $[\varepsilon]$ if determined from (6.1) is not the same as (6.2). $[\varepsilon]$ in (6.1) is a quadratic function of $\left[\sigma^{[0]}\right]$ and $\left[\sigma^{[0]}\right]$ in $(6.2)$ is a quadratic function of $[\varepsilon]$. In these forms $((6.1)$ and (6.2)), the material coefficients are not yet defined.

\subsubsection{Constitutive theories for $\sigma^{[0]}$ and $\varepsilon$ from entropy inequalities with material coefficients defined}

\section{(a) Based on integrity}

In this section we compare the constitutive theories for (6.1) and (6.2) after Taylor series expansion of the coefficients in (6.1) and (6.2) about a known configuration $\underline{\Omega}$, i.e. 
we consider (3.34) and (5.9), the most general case based on integrity.

$$
\begin{aligned}
& {[\varepsilon]=\left.\varepsilon_{0}\right|_{\Omega}[I]+{ }^{\varepsilon} b_{1}\left[\sigma^{[0]}\right]+{ }^{\varepsilon} b_{2}\left[\sigma^{[0]}\right]^{2}+} \\
& \sum_{j=1}^{3}{ }^{\varepsilon} a_{j}\left({ }_{\sim}^{\varepsilon} I^{j}[I]\right)+\sum_{j=1}^{3}{ }^{\varepsilon} c_{1 j}\left({ }_{\sim}^{\varepsilon} I^{j}\left[\sigma^{[0]}\right]\right)+\sum_{j=1}^{3}{ }^{\varepsilon} c_{2 j}\left({ }_{\sim}^{\varepsilon} I^{j}\left[\sigma^{[0]}\right]^{2}\right)+ \\
& { }^{\varepsilon} d_{1}\left(\theta-\theta_{\underline{\Omega}}\right)\left[\sigma^{[0]}\right]+{ }^{\varepsilon}{\underset{\sim}{d}}_{2}\left(\theta-\theta_{\underline{\Omega}}\right)\left[\sigma^{[0]}\right]^{2}-\left(\alpha_{t m}\right)_{\underline{\Omega}}\left(\theta-\theta_{\underline{\Omega}}\right)[I] \\
& {\left[\sigma^{[0]}\right]=\left.\sigma_{0}\right|_{\underline{\Omega}}[I]+\tilde{\sigma}_{1}[\varepsilon]+\tilde{\sigma}_{2}[\varepsilon]^{2}+} \\
& \sum_{j=1}^{3} \sigma \tilde{a}_{j}\left({ }_{\sim}^{\sigma} I^{j}[I]\right)+\sum_{j=1}^{3}{ }^{\sigma} \tilde{c}_{1 j}\left({ }_{\sim}^{\sigma} I^{j}[\varepsilon]\right)+\sum_{j=1}^{3}{ }^{\sigma} \tilde{c}_{2 j}\left({ }_{\sim}^{\sigma} I^{j}[\varepsilon]^{2}\right)+ \\
& \sigma \tilde{d}_{1}\left(\theta-\theta_{\underline{\Omega}}\right)[\varepsilon]+{ }^{\sigma} \tilde{d}_{2}\left(\theta-\theta_{\underline{\Omega}}\right)[\varepsilon]^{2}-\left(\tilde{\alpha}_{t m}\right)_{\underline{\Omega}}\left(\theta-\theta_{\underline{\Omega}}\right)[I]
\end{aligned}
$$

The material coefficients in (6.5) are functions of the combined invariants of $\boldsymbol{\sigma}^{[0]}, \boldsymbol{g}$ and $\theta$ whereas the material coefficients in (6.6) are functions of the combined invariants of $\boldsymbol{\varepsilon}$, $\boldsymbol{g}$ and $\theta$, all defined in a known configuration $\underline{\Omega}$. Lack of equivalence between these two theories is rather obvious.

\section{(b) Simplified form of the constitutive theories derived based on integrity}

We consider the most simplified form of the constitutive theories for $\boldsymbol{\sigma}^{[0]}$ and $\boldsymbol{\varepsilon}$ resulting from $\Psi$ and $\Phi$, i.e. we consider (3.35) and (5.10), which are linear in the components of $\boldsymbol{\sigma}^{[0]}$ and $\boldsymbol{\varepsilon}$, respectively.

$$
\begin{array}{lll}
{[\varepsilon]=\left.\varepsilon_{0}\right|_{\underline{\Omega}}[I]+{ }^{\varepsilon} b_{\sim}\left[\sigma^{[0]}\right]+\underline{\sim}_{a_{1}} \operatorname{tr}\left[\sigma^{[0]}\right][I]+\left(\alpha_{t m}\right)_{\underline{\Omega}}\left(\theta-\theta_{\underline{\Omega}}\right)[I] \quad:} & G \\
{\left[\sigma^{[0]}\right]=\left.\sigma_{0}\right|_{\underline{\Omega}}[I]+{ }^{\sigma} \tilde{b}_{1}[\varepsilon]+{ }^{\sigma} \tilde{a}_{1} \operatorname{tr}[\varepsilon][I]+\left(\tilde{\alpha}_{t m}\right)_{\underline{\Omega}}\left(\theta-\theta_{\underline{\Omega}}\right)[I] \quad:} & H
\end{array}
$$

If we assume that the material coefficients in (6.7) and (6.8) are constant, i.e. not functions of the invariants of $\boldsymbol{\sigma}^{[0]}$ and $\boldsymbol{\varepsilon}$, respectively, then the two theories are equivalent, i.e. $\left[\sigma^{[0]}\right]$ determined from (6.7) is the same as $\left[\sigma^{[0]}\right]$ in (6.8) and $[\varepsilon]$ determined from (6.8) is the 
same as $[\varepsilon]$ in (6.7). Thus, for linear elasticity between $\boldsymbol{\sigma}^{[0]}$ and $\boldsymbol{\varepsilon}$, these two theories are identical. We keep in mind that $\boldsymbol{\varepsilon}$ is a measure of finite strain.

\subsubsection{Constitutive theories for $\sigma^{[0]}$ and $\varepsilon$ from Taylor series expan- sions of $\Psi$ and $\Phi$}

In this case we first examine the most general form of the constitutive theories, i.e. we consider (3.26) and (5.5).

$$
\begin{array}{llll}
\varepsilon_{m n} & =\left.\varepsilon_{m n}^{0}\right|_{\underline{\Omega}}+\left.\underline{\underline{C}}_{m n i j}\right|_{\underline{\Omega}} \sigma_{i j}^{[0]}+\left.\underline{\underline{C}}_{m n i j k l}\right|_{\underline{\Omega}} \sigma_{i j}^{[0]} \sigma_{k l}^{[0]}+\ldots & : & G \\
\sigma_{m n}^{[0]} & =\left.\sigma_{m n}^{0}\right|_{\underline{\Omega}}+\left.\overline{\bar{C}}_{m n i j}\right|_{\underline{\Omega}} \varepsilon_{i j}+\left.\left(\underline{\bar{C}}_{m n i j k l}\right)\right|_{\underline{\Omega}} \varepsilon_{i j} \varepsilon_{k l}+\ldots & : & H
\end{array}
$$

Material coefficients in (6.9) are functions of $\left.\sigma^{[0]}\right|_{\underline{\Omega}}$ and $\left.\theta\right|_{\underline{\Omega}}$ whereas the material coefficients in (6.10) are functions of $\left.\varepsilon\right|_{\underline{\Omega}}$ and $\left.\theta\right|_{\underline{\Omega}}$. Furthermore, in (6.9) $\boldsymbol{\varepsilon}$ is a quadratic function of $\boldsymbol{\sigma}^{[0]}$, whereas in (6.10) $\boldsymbol{\sigma}^{[0]}$ is a quadratic function of $\boldsymbol{\varepsilon}$. Thus, there is no equivalence between these theories. In the most simplified case we assume $\boldsymbol{\varepsilon}$ to be a linear function of $\boldsymbol{\sigma}^{[0]}$ and $\boldsymbol{\sigma}^{[0]}$ to be a linear function of $\boldsymbol{\varepsilon}$, i.e. we consider

$$
\begin{array}{llll}
\varepsilon_{m n} & =\left.\varepsilon_{m n}^{0}\right|_{\underline{\Omega}}+\left.\underline{\underline{C}}_{m n i j}\right|_{\underline{\Omega}} \sigma_{i j}^{[0]} & : & G \\
\sigma_{m n}^{[0]}=\left.\sigma_{m n}^{0}\right|_{\underline{\Omega}}+\left.\overline{\bar{C}}_{m n i j}\right|_{\underline{\Omega}} \varepsilon_{i j} & : & H
\end{array}
$$

In these theories the material coefficients in (6.11) are functions of $\boldsymbol{\sigma}^{[0]}$ and $\theta$ and those in (6.12) are functions of $\boldsymbol{\varepsilon}$ and $\theta$ in the known configuration $\underline{\Omega}$. Thus, (6.11) and (6.12) are not precisely equivalent. If we assume the material coefficients to be constant, i.e. independent of deformation as in the case of linear elasticity, then (6.11) and (6.12) are equivalent, keeping in mind that $\boldsymbol{\varepsilon}$ is a strain measure for finite deformation. 


\subsection{Constitutive theories for heat vector $q$}

In this section we compare the constitutive theories for $\boldsymbol{q}$ derived using $\Psi$ and $\Phi$.

\subsubsection{Derived directly using conditions resulting from the entropy in-} equality

Regardless of $\Psi$ or $\Phi$, the entropy inequality requires that the heat vector satisfy

$$
q_{i} g_{i} \leq 0
$$

Thus, the constitutive theory derived directly using (6.13)

$$
q_{i}=-k_{i j}(\theta) g_{j}=-[k(\theta)]\{g\}
$$

is the same for $\Psi$ as well as $\Phi$.

\subsubsection{Using the theory of generators and invariants}

In this approach of deriving the constitutive theories for $\boldsymbol{q}$ we consider two approaches

(a) Approach I

In this case we choose the following

$$
\begin{array}{lll}
\boldsymbol{q}=\boldsymbol{q}(\boldsymbol{g}, \theta) & : & G \\
\boldsymbol{q}=\boldsymbol{q}(\boldsymbol{g}, \theta) & : & H
\end{array}
$$

Since in (6.15) and (6.16) the generators and invariants of the argument tensors of $\boldsymbol{q}$ are the same, the resulting constitutive theories from (6.15) and (6.16) are identical (Equations (3.50) or (5.14)) 


\section{(b) Approach II}

Here we consider

$$
\begin{aligned}
& \boldsymbol{q}=\boldsymbol{q}\left(\boldsymbol{\sigma}^{[0]}, \boldsymbol{g}, \theta\right) \quad: \quad G \\
& \boldsymbol{q}=\boldsymbol{q}(\boldsymbol{\epsilon}, \boldsymbol{g}, \theta) \quad: \quad H
\end{aligned}
$$

It is obvious that the combined generators and the invariants of the argument tensors of $\boldsymbol{q}$ in (6.17) and (6.18) are different so the resulting constitutive theories for $\boldsymbol{q}$ from (6.17) and (6.18) are different.

However, when there is equivalence (only for linear elasticity) between the constitutive theories for $[\varepsilon]$ and $\left[\sigma^{[0]}\right]$ resulting from $\Psi$ and $\Phi$ then the two constitutive theories for $\boldsymbol{q}$ resulting from (6.17) and (6.18) are equivalent. 


\section{Chapter 7}

\section{Summary and conclusions}

In this thesis we have presented constitutive theories for finite deformation of homogeneous, isotropic thermoelastic solids in Lagrangian description that are derived using the conditions resulting from the entropy inequality expressed in terms of Gibbs potential $\Psi$. The second Piola-Kirchoff stress tensor $\boldsymbol{\sigma}^{[0]}$ and Green's strain tensor $\boldsymbol{\varepsilon}$ are used as conjugate pairs. The condition resulting from the entropy inequality in $\Psi$ permit derivation of constitutive theory for $\boldsymbol{\varepsilon}$ in terms of $\boldsymbol{\sigma}^{[0]}$ and $\theta$. In this thesis we have presented three different approaches for deriving constitutive theories for $\boldsymbol{\varepsilon}$ : (i) assuming the Gibbs potential to be a function of the invariants of the conjugate stress tensor and then using the conditions resulting from the entropy inequality, (ii) using theory of generators and invariants, and (iii) expanding Gibbs potential in conjugate stress tensor using Taylor series about a known configuration and then using the conditions resulting from the entropy inequality.

The constitutive theories for $\varepsilon$ resulting from these three approaches are compared (3.1.3) for equivalence between them as well as their merits and shortcomings (3.1.4). The constitutive theories for heat vector have been derived: (i) directly using the condi- 
tions resulting from the entropy inequality, (ii) using $\boldsymbol{q}=\boldsymbol{q}(\boldsymbol{g}, \theta)$ in conjunction with the theory of generators and invariants and (iii) using $\boldsymbol{q}=\boldsymbol{q}\left(\boldsymbol{\sigma}^{[0]}, \boldsymbol{g}, \theta\right)$ and the theory of generators and invariants. These theories are compared for equivalence, their merits, and their shortcomings.

The constitutive theories derived using entropy inequality in Gibbs potential $\Psi$ are compared for equivalence with those resulting from the entropy inequality expressed in terms of Helmholtz free energy $\Phi$. Details are presented in chapter 6 and are not repeated again for brevity. It is shown that even in the most simplified constitutive theory for $\boldsymbol{q}$ using $\boldsymbol{q}=\boldsymbol{q}\left(\boldsymbol{\sigma}^{[0]}, \boldsymbol{g}, \theta\right)$ in conjunction with the theory of generators and invariants we demonstrate that the stress field influences heat conduction. Compression results in enhanced heat transfer whereas tension reduces effective heat transfer. In case of Helmholtz free energy density $\Phi$, a similar theory for $\boldsymbol{q}$ shows influence of the strain field on heat transfer in a similar fashion.

It is worth noting that entropy inequality expressed either in terms of $\Psi$ or $\Phi$ has no assumptions or approximations, i.e. entropy inequality in $\Psi$ is precisely equivalent to the entropy inequality in $\Phi$, yet the constitutive theories resulting from these two only show equivalence for extremely simplified cases. The major cause of this of course are the assumptions regarding arguments of $\boldsymbol{\sigma}^{[0]}$ in case of $\Phi$ and that of $\boldsymbol{\varepsilon}$ in case of $\Psi$. For example, we have $\boldsymbol{\varepsilon}=\boldsymbol{\varepsilon}\left(I_{\sigma^{[0]}}, \mathbb{I}_{\sigma^{[0]}}, \mathbb{I I}_{\sigma^{[0]}}, \theta\right)$ in $\Psi$ and $\boldsymbol{\sigma}^{[0]}=\boldsymbol{\sigma}^{[0]}\left(I_{\varepsilon}, \mathbb{I}_{\varepsilon}, \mathbb{I I}_{\varepsilon}, \theta\right)$ in $\Phi$. Since there is no equivalence between all invariants of $\boldsymbol{\sigma}^{[0]}$ and $\boldsymbol{\varepsilon}$, the equivalence in the resulting constitutive theories is lost as well.

This work demonstrates two equally effective parallel approaches for deriving constitutive theories for thermoelastic solids in Lagrangian description. In general, constitutive theory for $\boldsymbol{\sigma}^{[0]}$ in terms of $\boldsymbol{\varepsilon}$ resulting from $\Phi$ permits elimination of $\boldsymbol{\sigma}^{[0]}$ as a dependent 
variable from the mathematical models derived using conservation laws, thereby yielding a more compact mathematical model due to reduced number of dependent variables. This is beneficial in improving efficiency of numerical computations of the solutions of the evolutions described by the mathematical models. On the other hand, in the constitutive theories derived using $\Psi, \boldsymbol{\varepsilon}$ is a function of $\boldsymbol{\sigma}^{[0]}$ and $\theta$, hence in general these constitutive equations may not permit elimination of $\boldsymbol{\sigma}^{[0]}$ as dependent variables from the mathematical models. Besides this argument, the usefulness and the effectiveness of one theory over the other is dictated by the desired physics. 


\section{Bibliography}

[1] Surana, K. S. and Reddy, J. N. Continuum Mechanics. (Manuscript of the textbook in preparation, 2012).

[2] Eringen, A. C. Nonlinear Theory of Continuous Media. McGraw-Hill, 1962.

[3] Prager, W. Strain Hardening under Combined Stresses. Journal of Applied Physics, 16:837-840, 1945.

[4] Reiner, M. A Mathematical Theory of Dilatancy. American Journal of Mathematics, $67: 350-362,1945$.

[5] Rivlin, R. S. and Ericksen, J. L. Stress-Deformation Relations for Isotropic Materials. Journal of Rational Mechanics and Analysis, 4:323-425, 1955.

[6] Rivlin, R. S. Further Remarks on the Stress-Deformation Relations for Isotropic Materials. Journal of Rational Mechanics and Analysis, 4:681-702, 1955.

[7] Todd, J. A. Ternary Quadratic Types. Philosophical Transactions of the Royal Society of London. Series A: Mathematical and Physical Sciences, 241:399-456, 1948.

[8] Eringen, A. C. Mechanics of Continua. John Wiley and Sons, 1967. 
[9] Wang, C. C. On Representations for Isotropic Functions, Part I. Archive for Rational Mechanics and Analysis, 33:249, 1969.

[10] Wang, C. C. On Representations for Isotropic Functions, Part II. Archive for Rational Mechanics and Analysis, 33:268, 1969.

[11] Wang, C. C. A New Representation Theorem for Isotropic Functions, Part I and Part II. Archive for Rational Mechanics and Analysis, 36:166-223, 1970.

[12] Wang, C. C. Corrigendum to 'Representations for Isotropic Functions'. Archive for Rational Mechanics and Analysis, 43:392-395, 1971.

[13] Smith, G. F. On a Fundamental Error in two Papers of C.C. Wang, 'On Representations for Isotropic Functions, Part I and Part II'. Archive for Rational Mechanics and Analysis, 36:161-165, 1970.

[14] Smith, G. F. On Isotropic Functions of Symmetric Tensors, Skew-Symmetric Tensors and Vectors. International Journal of Engineering Science, 9:899-916, 1971.

[15] Spencer, A. J. M. and Rivlin, R. S. The Theory of Matrix Polynomials and its Application to the Mechanics of Isotropic Continua. Archive for Rational Mechanics and Analysis, 2:309-336, 1959.

[16] Spencer, A. J. M. and Rivlin, R. S. Further Results in the Theory of Matrix Polynomials. Archive for Rational Mechanics and Analysis, 4:214-230, 1960.

[17] Spencer, A. J. M. Theory of Invariants. Chapter 3 'Treatise on Continuum Physics, I' Edited by A. C. Eringen, Academic Press, 1971. 
[18] Boehler, J. P. On Irreducible Representations for Isotropic Scalar Functions. Journal of Applied Mathematics and Mechanics / Zeitschrift für Angewandte Mathematik und Mechanik, 57:323-327, 1977.

[19] Zheng, Q. S. On the Representations for Isotropic Vector-Valued, Symmetric TensorValued and Skew-Symmetric Tensor-Valued Functions. International Journal of Engineering Science, 31:1013-1024, 1993.

[20] Zheng, Q. S. On Transversely Isotropic, Orthotropic and Relatively Isotropic Functions of Symmetric Tensors, Skew-Symmetric Tensors, and Vectors. International Journal of Engineering Science, 31:1399-1453, 1993.

[21] Surana, K. S., Nunez, D., Reddy, J. N., and Romkes, A. Rate Constitutive Theory for Ordered Thermofluids. Continuum Mechanics and Thermodynamics (In print, 2012).

[22] Surana, K. S., Nunez D., Reddy, J. N., and Romkes, A. Rate Constitutive Theory for Ordered Thermoelastic Solids. Annals of Solid and Structural Mechanics (In print, 2012).

[23] Surana, K. S., Moody, T. and Reddy, J. N. Rate Constitutive Theories in Lagrangian Description for Thermoelastic Solids. Continuum Mechanics and Thermodynamics (In preparation, 2012).

[24] Gurtin, M. The Continuum Mechanics of Coherent Two-Phase Elastic Solids with Mass Transport. Proceedings of the Royal Society A: Mathematical, Physical, and Engineering Sciences, 440(1909):323-343, 1993. 
[25] Schapery, R. A. Application of Thermodynamics to Thermomechanical, Fracture, and Birefringent Phenomena in Viscoelastic Media. Journal of Applied Physics, 35(5):1451-1465, 1964.

[26] Shapiro, N. Z. and Shapley, L. S. Mass Action Laws and the Gibbs Free Energy Function. Journal of the Society for Industrial and Applied Mathematics, 13(2):353$375,1965$.

[27] Landel, R. F. and Peng, S. T. J. Equations of State and Constitutive Equations. Journal of Rheology, 30(4):741-765, 1986.

[28] Hassanizadeh, S. M. Mechanics and Thermodynamics of Multiphase Flow in Porous Media Including Interphase Boundaries. Advances in Water Resources, 13(4):169186, 1990.

[29] Stevens, R. N. and Guiu, F. Energy Balance Concepts in the Physics of Fracture. Proceedings of the Royal Society A: Mathematical, Physical, and Engineering Sciences, 435(1893):169-184, 1991.

[30] Lustig, S. R., Shay Jr., R. M. and Caruthers, J. M. Thermodynamic Constitutive Equations for Materials with Memory on a Material Time Scale. Journal or Rheology, 40(1), 1996.

[31] Maire, J. F. and Chaboche, J. L. A New Formulation of Continuum Damage Mechanics (CDM) for Composite Materials. Aerospace Science and Technology, 1(4):247257, 1997. 
[32] Gray, W. G. and Hassanizadeh, S. M. Macroscale Continuum Mechanics for Multiphase Porous-Media Flow Including Phases, Interfaces, Common Lines and Common Points. Advances in Water Resources, 21(4):261-281, 1998.

[33] O’Rielly, O. M. On Constitutive Relations for Elastic Rods. International Journal of Solids and Structures, 35(11):1009-1024, 1998.

[34] Fischer, F. D., Oberaigner, E. R., Tanaka, K. and Nishimura, F. Transformation Induced Plasticity Revised an Updated Formulation. International Journal of Solids and Structures, 35(18):2209-2227, 1998.

[35] Houlsby, G. T. and Puzrin, A. M. A Thermomechanical Framework for Constitutive Models for Rate-Independent Dissipative Materials. International Journal of Plasticity, 16(9):1017-1047, 2000.

[36] Houlsby, G. T. and Puzrin, A. M. Rate-Dependent Plasticity Models Derived From Potential Functions. Journal of Rheology, 46(1), 2002.

[37] Lubarda, V. A. On Thermodynamic Potentials in Linear Thermoelasticity. International Journal of Solids and Structures, 41(26):7377-7398, 2004.

[38] Zhao, J., Sheng, D. and Collins, I. F. Thermomechanical Formulation of Strain Gradient Plasticity for Geomaterials. Journal of Mechanics of Materials and Structures, 1(5), 2006.

[39] Rajagopal, K. R. and Srinivasa, A. R. A Gibbs-Potential-Based Formulation for Obtaining the Response Functions for a Class of Viscoelastic Materials. Proceed- 
ings of the Royal Society A: Mathematical, Physical and Engineering Sciences, 467(2125):39-58, 2010.

[40] Bridges, C. Implicit Rate-Type Models for Elastic Bodies: Development, Integration, Linearization \& Application. PhD thesis, Texas A\&M University, 2011. 